\title{
Tachyon condensation and quark mass in modified Sakai-Sugimoto model
}

\author{
Avinash Dhar ${ }^{\star}$ and Partha Nag ${ }^{\star}$ \\ * Tata Institute of Fundamental Research, Homi Bhabha Road, \\ Mumbai 400 005, India \\ ${ }^{\diamond}$ High Energy Accelerator Research Organization (KEK) \\ Tsukuba, Ibaraki 305-0801, Japan \\ adhar@theory.tifr.res.in, parthanag@theory.tifr.res.in
}

\begin{abstract}
This paper continues the investigation of the modified Sakai-Sugimoto model proposed in arXiv:0708.3233. Here we discuss in detail numerical solutions to the classical equations for the brane profile and the tachyon condensate. An ultraviolet cut-off turns out to be essential because the numerical solutions tend to rapidly diverge from the desired asymptotic solutions, beyond a sufficiently large value of the holographic coordinate. The required cut-off is determined by the non-normalizable part of the tachyon and is parametrically far smaller than that dictated by consistency of a description in terms of 10-dimensional bulk gravity. In arXiv:0708.3233 we had argued that the solution in which the tachyon field goes to infinity at the point where the brane and antibrane meet has only one free parameter, which may be taken to be the asymptotic brane-antibrane separation. Here we present numerical evidence in favour of this observation. We also present evidence that the non-normalizable part of the asymptotic tachyon solution, which is identified with quark mass in the QCD-like boundary theory, is determined by this parameter. We show that the normalizable part of the asymptotic tachyon solution determines the quark condensate, but this requires holographic renormalization of the on-shell boundary brane action because of the presence of infinite cut-off dependent terms. Our renormalization scheme gives an exponential dependence on the cut-off to the quark mass. We also discuss meson spectra in detail and show that the pion mass is nonzero and satisfies the Gell-Mann-Oakes-Renner relation when a small quark mass is switched on.
\end{abstract}

KEYwORDs: Chiral symmetry breaking, Holographic QCD, Gauge-gravity duality. 


\section{Contents}

1. Introduction 2

2. Modified Sakai-Sugimoto model with tachyon 4

2.1 Brane-antibrane pair with tachyon 5

2.2 Classical equations for brane profile and tachyon 7

2.3 Solution for large $u$

2.4 Quark mass and the ultraviolet cut-off 10

2.5 Solution for $u \sim u_{0}$

3. Numerical solutions 12

3.1 Verification of the UV and IR analytic solutions 13

3.2 Behaviour of the non-normalizable part 14

3.3 Behaviour of the asymptotic brane-antibrane separation 17

3.4 Comparison with the Sakai-Sugimoto solution 17

4. The chiral condensate 20

4.1 Action for $(u, x)$-dependent $T$ and $h \quad 21$

4.2 Condensate in terms of the tachyon solution 24

5. The meson spectra 26

5.1 Vector mesons 27

5.2 Axial vector and pseudoscalar mesons 28

5.3 Relation between pion mass and non-normalizable part of tachyon 32

6. Summary and Discussion 35

A. Overlapping $D 8-\bar{D} 8$-brane system 37

B. Scalar fluctuations 38

C. Calculation of the exact $(u, x)$-dependent action 43 


\section{Introduction}

The model of Sakai and Sugimoto (SS) [1] has been very successful in reproducing many of the qualitative features of non-abelian chiral symmetry breaking in QCD. In this model, the 'colour' Yang-Mills fields are provided by the massless open string fluctuations of a stack of a large number $N_{c}$ of $D 4$-branes, which are extended along the four space-time directions and in addition wrap a circle [2]. In the strong coupling limit, this stack of $D 4$-branes has a dual description in terms of a classical gravity theory. Flavour degrees of freedom are introduced in the probe approximation as fermionic open string fluctuations between the colour branes and

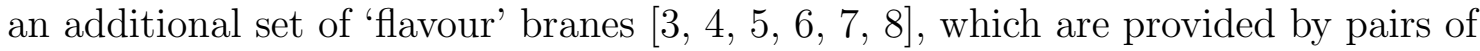
$D 8$ and $\overline{D 8}$-branes. In this setting, chiral symmetry breaking has a nice geometrical picture. In the ultraviolet, chiral symmetry arises on flavour $D 8$-branes and $\overline{D 8}$ branes, which are located at well-separated points on the circle, while they are extended along the remaining eight spatial directions, including the holographic radial direction. Chiral symmetry breaking in the infrared is signaled by a smooth joining of the flavour branes and antibranes at some point in the bulk.

Despite its many qualitative and some quantitative successes [1, 9, 10, 11, 12, 13, 14, 15, 16, 17, the SS model has some deficiencies: (i) It does not have parameters associated with quark mass and the chiral condensate. On the other hand, there is a parameter, the asymptotic separation between the flavour branes and antibranes, which, within the SS scenario, finds no counterpart in QCD; (ii) The SS model also ignores the open string tachyon between flavour $D 8$-brane and $\bar{D} 8$-brane, which may be reasonable in the ultraviolet where the branes and antibranes are well separated, but is not so at the place in the bulk where the branes join. It is often argued that in the curved background of the wrapped $D 4$-branes, the geometry forces flavour branes to join in the interior. While this is true of flavour branes and antibranes that are well-separated asymptotically (separation of the order of the antipodal distance), it cannot be the reason when the separation is small and the branes and antibranes meet far away from the central region. For small separation, the effective radius of the direction on which the $D 4$-branes are wrapped is very large and so one would expect tachyon condensation to be the primary reason for branes and antibranes meeting, as in the extremal D4-brane metric. Since the tachyon field takes an infinitely large value in the true ground state ${ }^{1}$, the perturbative stability argument given in [1], valid for small fluctuations of the tachyon field near the local minimum at the origin, does not apply.

It has recently been suggested in [19, 20, 21] that tachyon condensation on a brane-antibrane system describes the physics of chiral symmetry breaking in a better and more complete way. If the brane and antibrane are well-separated [20, 21] then one also retains the nice geometric picture of the SS model for non-

\footnotetext{
${ }^{1}$ For a recent review of this subject, see [18].
} 
abelian chiral symmetry breaking. The purpose of the present work is to complete the investigations started in [21]. Here we give detailed numerical solutions to the classical equations for the brane profile and the tachyon. We show that the solution in which the tachyon diverges at the point in the bulk where the brane and antibrane meet has only one free parameter, which may be taken to be the asymptotic separation between the flavour brane and the antibrane. We present numerical evidence that the non-normalizable part of the asymptotic tachyon solution is determined by this parameter. Thus, by the usual dictionary of AdS/CFT [22, 23, 24, 25], this parameter determines quark mass in the boundary theory [26, [16]. The parameter for the asymptotic brane-antibrane separation is present in the SS model also, but in that setting it cannot be explained as a parameter in QCD. Thus this parameter, which seems mysterious in the SS setting, finds a natural explanation in our model. The presence of a non-normalizable part in the tachyon solution necessitates introduction of an ultraviolet cut-off. This is because in this case the numerical solutions tend to rapidly diverge from the desired asymptotic solutions, beyond a sufficiently large value of the radial coordinate, determined by the magnitude of the non-normalizable part. This cut-off is parametrically far smaller than the cut-off of order $N^{4 / 3}$ expected because of the breakdown of description in terms of a 10-dimensional gravity theory. Removing the cut-off, therefore, necessarily involves tuning the non-normalizable part to zero. We discuss how this should be done appropriately. We also discuss the chiral condensate and its determination by the normalizable part of the asymptotic tachyon solution. This determination is subtle for two reasons. One is the fact that the space-time independent classical solutions are described by a single parameter and hence the non-normalizable part of the tachyon cannot be varied independent of the other parameters. The resolution of this issue requires us to consider more general solutions by incorporating space-time dependence. But for this one has to go beyond the expansion in small space-time dependent fluctuations around space-time independent solutions, basically because this expansion is singular for the tachyon solution in the infrared. An exact space-time dependent action is needed, which we derive. The other subtlety has to do with the necessity of an ultraviolet cut-off. To extract cut-off independent physics, we add counter-terms to the $D 8$-brane action to remove terms in the boundary action which are divergent as the cut-off is formally allowed to go to infinity. With an appropriate choice of the counter-terms we get a finite value for the chiral condensate. Finally, we discuss meson spectra in detail and show that the pion mass is nonzero in the presence of a non-normalizable part of the tachyon and that it satisfies the Gell-Mann-Oakes-Renner (GOR) relation when quark mass is small.

The organization of this paper is as follows. In the next section we will briefly review the essential features of the modified SS model with the tachyon present. This section also includes a more detailed discussion of the cut-off and its implications 
than given in [21]. In section 3 we describe in detail the numerical solutions for the brane profile and the tachyon. This section also contains a discussion of the parameters of the solutions and their determination in terms of a single parameter, namely, the asymptotic brane-antibrane separation. In section 1 we discuss the subtleties involved in deriving an expression for the chiral condensate in terms of the parameters of the solutions. We derive the exact 5-dimensional action in which the tachyon and brane-antibrane separation fields have dependence on space-time as well as the holographic coordinate and discuss solutions to the equations derived from this action. We also discuss the counter-terms required to make the chiral condensate finite as the cut-off is formally removed to infinity. In section 5 we analyse small fluctuations around the classical solution for the meson spectra. We show that the existence of a massless pion is guaranteed if the non-normalizable part of the tachyon solution vanishes. For a non vanishing non-normalizable part of the tachyon solution, we obtain an expression for the pion mass and derive the GOR relation for it. We end with a summary and discussion in section 6. The Appendices contain details of some calculations.

As this work was nearing completion, the works [28] and [29] appeared which have discussed the problem of quark mass in SS model using different methods.

\section{Modified Sakai-Sugimoto model with tachyon}

The Yang-Mills part of the SS model is provided by the near horizon limit of a set of $N_{c}$ overlapping $D 4$-branes, filling the $(3+1)$-dimensional space-time directions $x^{\mu}(\mu=1,2,3$ and 0$)$ and wrapping a circle in the $x^{4}$ direction of radius $R_{k}$. Anti periodic boundary condition for fermions on this circle gives masses to all fermions at the tree level (and scalars at one-loop level) and breaks all supersymmetries. At low energies compared to $l_{s}^{-1}$, the theory on the $D 4$-branes is $(4+1)$-dimensional pure Yang-Mills with 't Hooft coupling $\lambda_{5}=(2 \pi)^{2} g_{s} l_{s} N_{c}$, of length dimension. At energies lower than the Kaluza-Klein mass scale, $R_{k}^{-1}$, this reduces to pure YangMills in $(3+1)$ dimensions. This is true in the weak coupling regime, $\lambda_{5}<<R_{k}$, in which the dimensionally transmuted scale developed in the effective Yang-Mills theory in $(3+1)$ dimensions is much smaller than the Kaluza-Klein mass scale, which is the high energy cut-off for the effective theory. In the strong coupling regime, $\lambda_{5}>>R_{k}$, in which the dual gravity description is reliable, these two scales

are similar. Therefore in this regime there is no separation between the masses of glueballs and Kaluza-Klein states. This is one of the reasons why the gravity regime does not describe real QCD, but the belief is that qualitative features of QCD like confinement and chiral symmetry breaking, which are easy to study in the strong coupling regime using dual geometry, survive tuning of the dimensionless parameter $\lambda_{5} / R_{k}$ to low values. 
Flavours are introduced in this setting by placing a stack of $N_{f}$ overlapping $D 8$-branes at the point $x_{L}^{4}$ and $N_{f} \overline{D 8}$-branes at the point $x_{R}^{4}$ on the thermal circle. Massless open strings between $D 4$-branes and $D 8$-branes, which are confined to the $(3+1)$-dimensional space-time intersection of the branes, provide $N_{f}$ left-handed flavours. Similarly, massless open strings between $D 4$-branes and $\overline{D 8}$-branes provide an equal number of right-handed flavours, leading to a local $U\left(N_{f}\right)_{L} \times U\left(N_{f}\right)_{R}$ chiral gauge symmetry on the flavour $D 8$ and $\overline{D 8}$-branes. This chiral gauge symmetry is seen in the boundary theory as a global chiral symmetry.

In the large $N_{c}$ and strong coupling limit, the appropriate description of the wrapped $D 4$-branes is given by the dual background geometry. This background solution can be obtained from the Euclidean type IIA sugra solution for nonextremal $D 4$-branes by a wick rotation of one of the four noncompact directions which the $D 4$-branes fill, in addition to wrapping the compact (temperature) direction. In the near horizon limit, it is given by [2, 27]

$$
\begin{aligned}
d s^{2} & =\left(\frac{U}{R}\right)^{3 / 2}\left(\eta_{\mu \nu} d x^{\mu} d x^{\nu}+f(U)\left(d x^{4}\right)^{2}\right)+\left(\frac{R}{U}\right)^{3 / 2}\left(\frac{d U^{2}}{f(U)}+U^{2} d \Omega_{4}^{2}\right), \\
e^{\phi} & =g_{s}\left(\frac{U}{R}\right)^{3 / 4}, \quad F_{4}=\frac{2 \pi N_{c}}{V_{4}} \epsilon_{4}, \quad f(U)=1-\frac{U_{k}^{3}}{U^{3}},
\end{aligned}
$$

where $\eta_{\mu \nu}=\operatorname{diag}(-1,+1,+1,+1)$ and $U_{k}$ is a constant parameter of the solution ${ }^{2}$. $R$ is related to the 5-d Yang-Mills coupling, $\lambda_{5}$, which is kept fixed in the decoupling limit, by $R^{3}=\frac{\lambda_{5} \alpha^{\prime}}{4 \pi}$. Also, $d \Omega_{4}, \epsilon_{4}$ and $V_{4}=8 \pi^{2} / 3$ are respectively the line element, the volume form and the volume of a unit $S^{4}$.

The above metric has a conical singularity at $U=U_{k}$ in the $U-x^{4}$ subspace which can be avoided only if $x^{4}$ has a specific periodicity. This condition relates the radius of the circle in the $x^{4}$ direction to the parameters of the background by

$$
R_{k}=\frac{2}{3}\left(\frac{R^{3}}{U_{k}}\right)^{\frac{1}{2}}
$$

For $\lambda_{5}>>R_{k}$ the curvature is small everywhere and so the approximation to a classical gravity background is reliable. As discussed in [27], at very large values of $U$, the string coupling becomes large and one has to lift the background over to the 11-dimensional M-theory description.

\subsection{Brane-antibrane pair with tachyon}

The effective field theory describing the dynamics of a brane-antibrane pair in a background geometry ${ }^{3}$ with the tachyon included has been discussed in [31, 32].

\footnotetext{
${ }^{2}$ Note that $U$ has dimension of length and is related to the energy scale $\tilde{U}$, which is kept fixed in the decoupling limit, by $U=\tilde{U} \alpha^{\prime}$.

${ }^{3}$ For simplicity, we will discuss the case of a single flavour, namely one brane-antibrane pair. Generalization to the multi-flavour case can be done using the symmmetrized trace prescription of 30 .
} 
The simplest case occurs when the brane and antibrane are on top of each other since in this case all the transverse scalars are set to zero. This is the situation considered in 19. However, in this configuration one loses the nice geometrical picture of chiral symmetry breaking of the SS model. The geometrical picture is retained in the case considered in [20, 21] where the brane and antibrane are separated in the compact $x^{4}$ direction. This requires construction of an effective tachyon action on a braneantibrane pair, taking into account the transverse scalars. Such an effective action with the brane and antibrane separated along a noncompact direction has been proposed in 31, 32 ${ }^{4}$. A generalization of this action to the case when the brane and antibrane are separated along a periodic direction is not known. However, for small separation $l(U)$ compared to the radius $R_{k}$ of the circle, the action in [32] should provide a reasonable approximation to the compact case. In the following we will assume this to be so. Then, the effective low-energy tachyon action for a $D 8$ and $\overline{D 8}$-brane pair for $l(U)<<R_{k}$ is given, in the above background, by ${ }^{5}$

$$
\begin{aligned}
S= & -\int d^{9} \sigma V(T, l) e^{-\phi}\left(\sqrt{-\operatorname{det} \mathrm{A}_{\mathrm{L}}}+\sqrt{-\operatorname{det} \mathrm{A}_{\mathrm{R}}}\right) \\
\left(A_{i}\right)_{a b}= & \left(g_{M N}-\frac{T^{2} l^{2}}{2 \pi \alpha^{\prime} Q} g_{M 4} g_{4 N}\right) \partial_{a} x_{i}^{M} \partial_{b} x_{i}^{N}+2 \pi \alpha^{\prime} F_{a b}^{i}+\frac{1}{2 Q}\left(2 \pi \alpha^{\prime}\left(D_{a} \tau\left(D_{b} \tau\right)^{*}+\left(D_{a} \tau\right)^{*} D_{b} \tau\right)\right. \\
& \left.+i l\left(g_{a 4}+\partial_{a} x_{i}^{4} g_{44}\right)\left(\tau\left(D_{b} \tau\right)^{*}-\tau^{*} D_{b} \tau\right)+i l\left(\tau\left(D_{a} \tau\right)^{*}-\tau^{*} D_{a} \tau\right)\left(g_{4 b}-\partial_{b} x_{i}^{4} g_{44}\right)\right),
\end{aligned}
$$

where

$$
Q=1+\frac{T^{2} l^{2}}{2 \pi \alpha^{\prime}} g_{44}, \quad D_{a} \tau=\partial_{a} \tau-i\left(A_{L, a}-A_{R, a}\right) \tau, \quad V(T, l)=g_{s} V(T) \sqrt{Q} .
$$

$T=|\tau|, i=L, R$ and we have used the fact that the background does not depend on $x^{4}$. The complete action also includes terms involving Chern-Simons (CS) couplings of the gauge fields and the tachyon to the RR background sourced by the $D 4$-branes. These will not be needed in the following analysis and hence have not been included here.

The potential $V(\tau)$ depends only on the modulus $T$ of the complex tachyon $\tau$. It is believed that $V(\tau)$ satisfies the following general properties [18]:

- $V(T)$ has a maximum at $T=0$ and a minimum at $T=\infty$ where it vanishes.

\footnotetext{
${ }^{4}$ Also see [33].

${ }^{5}$ Strictly speaking, this action is valid only when the brane and antibrane are separated along a noncompact direction. However, as we shall see later, a posteriori justification for using this action is provided by the classical solutions for the brane-antibrane profile. In these solutions, for small asymptotic separation, the brane and antibrane meet far away from the central region. In this case, to a good approximation, the factor $f(U)$ in the background metric can be set to identity, which is equivalent to setting the radius $R_{k}$ to infinity.
} 
- The normalization of $V(T)$ is fixed by the requirement that the vortex solution on the brane-antibrane system should produce the correct relation between $D p$ and $D(p-2)$-brane tensions. In the present case this means $V(0)=\mathcal{T}_{8}=1 /(2 \pi)^{8} l_{s}^{9} g_{s}$, the $D 8$-brane tension.

- In flat space, the expansion of $V(T)$ around $T=0$ up to terms quadratic in $T$ gives rise to a tachyon with mass-squared equal to $-1 / 2 \alpha^{\prime}$.

There are several proposals for $V(T)$ which satisfy these requirements [18], although no rigorous derivation exists. Examples are (i) the potential used in [34, 35, 36] for calculation of decay of unstable D-branes in two-dimensional string theory

$$
V(T)=\mathcal{T}_{8} \operatorname{sech} \sqrt{\pi} T
$$

and (ii) the potential obtained using boundary string field theory computation [37, 38, 39, 40]

$$
V(T)=\mathcal{T}_{8} e^{-\frac{\pi}{2} T^{2}} .
$$

Both these potentials satisfy the properties listed above. Note that the asymptotic form of the potential in (2.5) for large $T$ is $\sim e^{-\sqrt{\pi} T}$. The linear growth of the exponent with $T$ should be contrasted with the quadratic growth for the potential in (2.6). This difference will turn out to be important for the background tachyon solutions, which are discussed next.

We end this subsection with the following observation. It can be easily seen that in the decoupling limit all factors of $\alpha^{\prime}$ scale out of the entire action, without requiring any scaling of the transverse scalar $l$ or the tachyon $\tau$. In fact, the entire action can be rewritten in terms of $\lambda_{5}$ and $\tilde{U}$, quantities that are kept fixed in the scaling limit. Henceforth, we will use the convention $2 \pi \alpha^{\prime}=1$.

\subsection{Classical equations for brane profile and tachyon}

We will now look for an appropriate classical solution of the brane-antibrane-tachyon system. Let us set the gauge fields and all but the derivatives with respect to $U$ of $T$ and $x_{i}^{4}$ to zero. Moreover, we choose $x_{L}^{4}=l / 2$ and $x_{R}^{4}=-l / 2$ so that the separation between the brane and antibrane is $l$. In this case, in the static gauge the action (2.3) simplifies to ${ }^{6}$

$$
S=-V_{4} \int d^{4} x \int d U V(T)\left(\frac{U}{R}\right)^{-3 / 4} U^{4}\left(\sqrt{D_{L, T}}+\sqrt{D_{R, T}}\right),
$$

where $D_{L, T}=D_{R, T} \equiv D_{T}$ and

$$
D_{T}=f(U)^{-1}\left(\frac{U}{R}\right)^{-3 / 2}+f(U)\left(\frac{U}{R}\right)^{3 / 2} \frac{l^{\prime}(U)^{2}}{4}+T^{\prime}(U)^{2}+T(U)^{2} l(U)^{2} .
$$

\footnotetext{
${ }^{6}$ The CS term in the action does not contribute for such configurations.
} 
It is convenient to remove the dependence on $R$ (except for an overall factor in the action) through a redefinition of variables,

$$
U=u / R^{3}, \quad l(U)=R^{3} h(u), \quad U_{k}=u_{k} / R^{3} .
$$

In terms of the new variables, we get

$$
S=-V_{4} R^{-9} \int d^{4} x \int d u u^{13 / 4} V(T)\left(\sqrt{d_{L, T}}+\sqrt{d_{R, T}}\right)
$$

where

$$
d_{L, T}=d_{R, T} \equiv d_{T}=f(u)^{-1} u^{-3 / 2}+f(u) u^{3 / 2} \frac{h^{\prime}(u)^{2}}{4}+T^{\prime}(u)^{2}+T(u)^{2} h(u)^{2},
$$

with $f(u)=\left(1-u_{k}^{3} / u^{3}\right)$.

The effective potential for the tachyon can be obtained from this action by setting $T^{\prime}=h^{\prime}=0$. It is

$$
V_{\text {eff }}(T, l) \sim \operatorname{sech} \sqrt{\pi} T \sqrt{1+u^{3 / 2} T^{2} h^{2}}
$$

In Figure 11 we have plotted $V_{\text {eff }}$ as a function of $T$ for various values of $u$. We see that a perurbatively stable minimum at $T=0$ for large values of $u$ turns into an unstable maximum at a sufficiently small value of $u$. This is true for any fixed, nonzero value of $h$. Moreover, the value of $u$ at which there is an unstable maximum at $T=0$ increases as $h$ decreases.

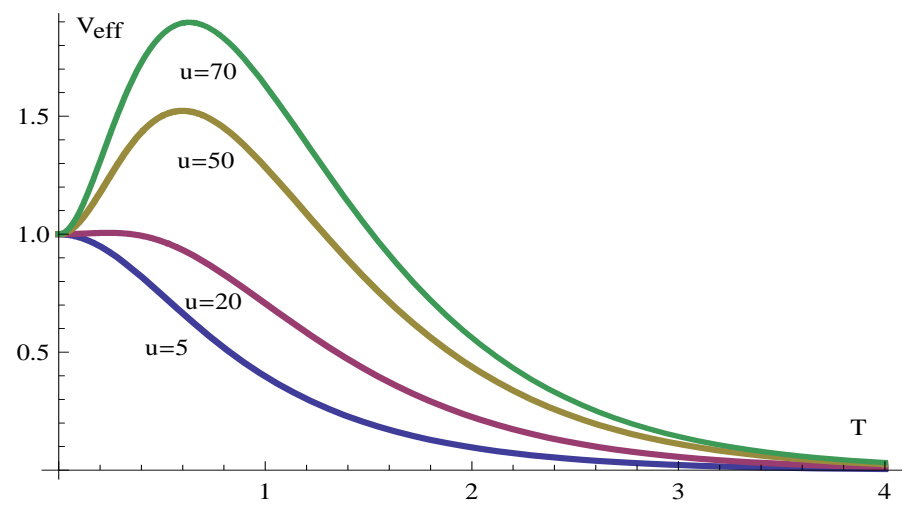

Figure 1: The effective potential $V_{\text {eff }}$ as a function of $T$ for different values of $u$ for a fixed non-zero value of $h$.

The equations of motion obtained from the action (2.10) are

$$
\begin{gathered}
\left(\frac{u^{\frac{13}{4}}}{\sqrt{d_{T}}} T^{\prime}(u)\right)^{\prime}=\frac{u^{\frac{13}{4}}}{\sqrt{d_{T}}}\left[T(u) h(u)^{2}+\frac{V^{\prime}(T)}{V(T)}\left(d_{T}-T^{\prime}(u)^{2}\right)\right], \\
\left(\frac{u^{\frac{13}{4}}}{\sqrt{d_{T}}} \frac{f(u)}{4} u^{\frac{3}{2}} h^{\prime}(u)\right)^{\prime}=\frac{u^{\frac{13}{4}}}{\sqrt{d_{T}}}\left[T(u)^{2} h(u)-\frac{V^{\prime}(T)}{V(T)} \frac{f(u)}{4} u^{\frac{3}{2}} h^{\prime}(u) T^{\prime}(u)\right] .
\end{gathered}
$$


Note that the 'prime' on $V(T)$ denotes a derivative w.r.t. its argument $T$ and not a derivative w.r.t. $u$.

This is a complicated set of coupled nonlinear differential equations which can be solved completely only numerically. To get some insight into the kind of solutions that are possible, however, we had analysed these equations in [21] for large $u$ and for $u$ near the brane-antibrane joining point in the bulk. For these values of $u$ the equations simplify and can be treated analytically. For the sake of completeness, we will summarize the results of this analysis here before proceeding to describe numerical solutions to these equations. As in the case without the tachyon, we are looking for solutions in which the brane and antibrane have a given asymptotic separation $h_{0}$, i.e. $h(u) \rightarrow h_{0}$ as $u \rightarrow \infty$, and they join at some interior point in the bulk, i.e. $h(u) \rightarrow 0$ at $u=u_{0} \geq u_{k}{ }^{7}$. Moreover, we want the tachyon (i) to vanish as $u \rightarrow \infty$ so that the chiral symmetry is intact in the ultraviolet region and (ii) to go to infinity as $u$ approaches $u_{0}$ so that the QCD chiral anomalies are reproduced correctly [19].

\subsection{Solution for large $u$}

Here we seek a solution in which $h(u)$ approaches a constant $h_{0}$ and $T$ becomes small as $u \rightarrow \infty$. For small $T$ one can approximate $V^{\prime} / V \sim-\pi T^{8}$. If $T$ and $h^{\prime}$ go to zero sufficiently fast as $u \rightarrow \infty$ such that to the leading order one might approximate $d_{T} \sim u^{-3 / 2}$, then (2.13) can be approximated to

$$
\left(u^{4} T^{\prime}(u)\right)^{\prime}=h_{0}^{2} u^{4} T \text {. }
$$

The general solution of this equation is

$$
T(u)=\frac{1}{u^{2}}\left(T_{+} e^{-h_{0} u}+T_{-} e^{h_{0} u}\right) .
$$

In writing this solution we have ignored a higher order term in $1 / u$ for consistency with other terms in equation (2.13) that we have neglected at large $u$. We will discuss consistency of this solution below. Let us first discuss the solution for $h(u)$.

The fact that the tachyon takes small values for large $u$ makes it irrelevant for the leading asymptotic behaviour of $h$, which can be extracted from (2.14) by setting the r.h.s. to zero. The resulting equation is

$$
\left(u^{\frac{11}{2}} h^{\prime}(u)\right)^{\prime}=0
$$

which has the solution

$$
h(u)=h_{0}-h_{1} u^{-9 / 2} .
$$

${ }^{7}$ The inequality results from the lower bound on $u$.

${ }^{8}$ This follows from the general properties of the potential discussed in section 2.1. 
Here $h_{1}$ is restricted to positive values so that the branes come together in the bulk. For SS model without the tachyon, $h_{1}=\frac{4}{9} u_{0}^{4} f_{0}^{1 / 2}$, where $f_{0}=f\left(u_{0}\right), u_{0}$ being the value of $u$ where the branes meet in the bulk.

It is easy to convince oneself that the only solution to equations (2.13) and (2.14) in which $T$ vanishes asymptotically and $h$ goes to a constant is (2.16) with $T_{-}=0$. In particular, for example, these equations have no solutions in which $T$ vanishes asymptotically as a power law.

\subsection{Quark mass and the ultraviolet cut-off}

In the tachyon solution (2.16), the exponentially falling part satisfies the approximations under which (2.15) was derived for any large value of $u$. The exponentially rising part will, however, eventually become large and cannot be selfconsistently used. This is because for sufficiently large $u$, there is no consistent solution for $T$ which grows exponentially or even as a power-law to the original equations (2.13) and (2.14), if we impose the restriction that $h(u)$ should go to a constant asymptotically. This puts a restriction on the value of $u$ beyond which the generic solution (2.16) cannot be used. The most restrictive condition comes from the approximation $d_{T} \sim u^{-3 / 2}$. This requires the maximum value, $u_{\max }$, to satisfy the condition

$$
T_{+}^{2} e^{-2 h_{0} u_{\max }}+T_{-}^{2} e^{2 h_{0} u_{\max }}<<\frac{u_{\max }^{5 / 2}}{2 h_{0}^{2}}
$$

For generic values of $\left|T_{ \pm}\right|$and $h_{0}$, this inequality determines a range of values of $u_{\max }$ for which the solution (2.16) can be trusted. The value $T_{-}=0$ is special since in this case there is no upper limit on $u_{\max }$, except the cut-off that comes from the fact that the 10-dimensional description of the background geometry breaks down beyond some very large value $\left(\sim N_{c}^{4 / 3}\right)$ of $u$. However, as is clear from (2.19), for nonzero $\left|T_{-}\right|$one needs to choose a much smaller value of $u_{\max }$. Numerical calculations reported in the next section bear out this expectation.

It is important to emphasize that the ultraviolet cut-off we are talking about here does not merely play the usual role of a cut-off needed in any example of AdS/CFT with a non-normalizable part present in a solution to the bulk equations. The point is that there is no growing solution to the tachyon equation in the ultraviolet which is consistent with a brane profile that goes to a finite asymptotic brane-antibrane separation. This constraint limits the value of $u$ up to which the asymptotic solutions, (2.16) and (2.18), can be trusted.

One way to think about the inequality (2.19) is the following. Suppose for given values of $\left|T_{ \pm}\right|$we have chosen the largest value of $u_{\max }$ consistent with (2.19). Increasing $u_{\max }$ further would then be possible only if $\left|T_{-}\right|$is decreased appropriately, while $\left|T_{+}\right|$can be kept fixed, as $u_{\max }$ is increased. To be concrete, let us keep $\left|T_{+}\right|$ and $\left|T_{-}\right| e^{h_{0} u_{\max }}$ fixed as $u_{\max }$ is increased. The process of "removing the cut-off" can then be understood as increasing $u_{\max }$ and simultaneous decreasing $\left|T_{-}\right|$while 
keeping $\left|T_{+}\right|$and the combination $\left|T_{-}\right| e^{h_{0} u_{\max }}$ fixed. In this process, at some point $\left|T_{+}\right| e^{-h_{0} u_{\max }}$ would become much smaller than $\left|T_{-}\right| e^{h_{0} u_{\max }}$. As we shall see in the next section, however, limitations due to numerical accuracy prevent us from tuning $\left|T_{-}\right|$to very small values, or equivalently tuning $u_{\max }$ to be very large. Thus we are numerically restricted to rather small values of $u_{\max }$. For values of $u$ larger than $u_{\max }$, the inequality (2.19) breaks down and consequently the asymptotic solution (2.16) is not applicable. Clear evidence for this breakdown is seen in the numerical calculations reported in the next section.

It is natural to associate $T_{-}$with the quark mass since this parameter comes with the growing solution. Evidence for this will be given in section 5 where we will show that for a small nonzero value of this parameter, the pion mass is nonzero and proportional to it. It is also natural to associate $T_{+}$with the chiral condensate because it comes with the normalizable solution. It turns out that this association too is consistent, though this part of the story is somewhat more complicated, as we shall see in section t.

It is interesting to mention here that keeping the combination $\left|T_{-}\right| e^{h_{0} u_{\max }}=\rho$ fixed as the cut-off becomes large implies an exponential dependence of $\left|T_{-}\right|$on the $u_{\max }$, i.e. $\left|T_{-}\right|=\rho e^{-h_{0} u_{\max }}$. A similar dependence of the quark mass on the cut-off has been observed in [28, 29], though the methods used for computing quark mass in these works are quite different from ours. In [29] the cut-off arises from the location of a $D 6$-brane, which is additionally present in that model, thereby giving a physical meaning to the cut-off.

\subsection{Solution for $u \sim u_{0}$}

Here we are looking for a solution in which $h \rightarrow 0$ and $T \rightarrow \infty$ as $u \rightarrow u_{0}$. Let us assume a power law ansatz, namely

$$
h(u) \sim\left(u-u_{0}\right)^{\alpha}, \quad T(u) \sim\left(u-u_{0}\right)^{-\beta} .
$$

For a smooth joining of the brane and antibrane at $u_{0}$, the derivative of $h$ must diverge at this point, which is ensured if $\alpha<1$. Since for this ansatz $T^{2}$ is the largest quantity for $u \rightarrow u_{0}$, we can approximate $d_{T} \sim T^{\prime}(u)^{2}$. We will also need the asymptotic form of the potential $V(T)$ for large $T$, which depends on the specific potential being used. From the asymptotic form of the potential in (2.5), we get $V^{\prime}(T) / V(T) \sim-\sqrt{\pi}$, while for the potential in (2.6), we get $V^{\prime}(T) / V(T) \sim-\pi T$. Putting all this in (2.13) and (2.14), it is easy to verify that these equations cannot be satisfied by the ansatz (4.9) for the potential (2.6). They are, however, satisfied for the potential in (2.5). In fact, in this case the powers as well as the coefficients all get fixed ${ }^{9}$ :

$$
h(u)=\sqrt{\frac{26}{\pi u_{0} f_{0}}} u_{0}^{-3 / 4}\left(u-u_{0}\right)^{1 / 2}+\cdots,
$$

\footnotetext{
${ }^{9}$ In [20] the power of $\left(u-u_{0}\right)$ with which the brane-anibrane separation falls-off in the bulk has been left undetermined. This power is actually determined by (2.13) and (2.14), as can be easily
} 


$$
T(u)=\frac{\sqrt{\pi}}{4} f_{0} u_{0}^{3 / 2}\left(u-u_{0}\right)^{-2}+\cdots
$$

An important feature of the above solution is that it depends only on a single parameter, namely the value of $u_{0}$. We have checked that this feature persists in the next few higher orders in a power series expansion in $\left(u-u_{0}\right)$. This is in sharp contrast to the asymptotic solution (2.16), (2.18) which depends on all the four expected parameters, $T_{+}, T_{-}, h_{0}, h_{1}$. This reduction in the number of parameters is similar to what happens in the SS model where the solution for $u \sim u_{0}$ depends only on one parameter, although the asymptotic solution depends on two parameters. In the present case the reduction in the number of parameters is even more severe; the solution for $u \sim u_{0}$ matches with only a one-parameter subspace of the four-parameter space of asymptotic solutions. As we will discuss later, this one-parameter freedom of the classical solution turns out to be analogous to the freedom to add a bare quark mass in QCD.

For completeness, we note that there exists another solution in which $T$ does not diverge but goes to a nonzero constant as $u \rightarrow u_{0}$. In this case we can approximate $d_{T} \sim f(u) u^{3 / 2} h^{\prime}(u)^{2} / 4$. Substituting in (2.13) we see that the 1.h.s. diverges as $\left(u-u_{0}\right)^{-\alpha}$. The first term on the r.h.s. vanishes as a positive power, but the second term diverges as $\left(u-u_{0}\right)^{\alpha-1}$, since $\alpha<1$. For consistency we must have $\alpha=1 / 2$. The resulting solution

$$
\begin{aligned}
& h(u)=\frac{4}{u_{0}}\left(f_{0}\left(5 f_{0}+3\right)\right)^{-1 / 2}\left(u-u_{0}\right)^{1 / 2}+\cdots \\
& T(u)=t_{0}+\frac{2 u_{0}^{-1 / 2}}{\left(5 f_{0}+3\right)} \frac{V^{\prime}\left(u_{0}\right)}{V\left(u_{0}\right)}\left(u-u_{0}\right)+\cdots
\end{aligned}
$$

also satisfies (2.14). Note that no special condition was required for the tachyon potential to get this solution; this solution exists for any potential.

\section{Numerical solutions}

The equations (2.13), (2.14) cannot be solved analytically. One needs to use numerical tools to get a solution. We have made use of mathematica for this. Also, for numerical calculations we have chosen the potential (2.5), since there is no diverging solution for $T(u)$ for $u \sim u_{0}$ for the potential (2.6), as discussed above.

The numerical calculations are easier to do if we start from the $u=u_{0}$ end and evolve towards the large $u$ end. This avoids the fine-tuning one would have to do if one were to start from large values of $u$, where the general solution has four parameters, and end on a one-parameter subspace for $u \sim u_{0}$. We must

checked by consistently expanding these equations on both sides and going beyond the leading order in powers of $\left(u-u_{0}\right)$. We have also verified this power by numerical calculations reported in the next section. 
also satisfy the requirement of working in the parameter region of the background geometry corresponding to the strong coupling. In addition, we need to ensure that the asymptotic separation between flavour branes and antibranes is small compared to the radius of the $x^{4}$ circle. Mathematically, these requirements are $\lambda_{5}=8 \pi^{2} R^{3} \gg 2 \pi R_{k}$ and $l_{0} \ll \pi R_{k}$. Using (2.2) and (2.9), one gets $R^{3}=\frac{3}{2} R_{k} \sqrt{u_{k}}$. Then, these requirements become $\frac{1}{36 \pi^{2}} \ll u_{k} \ll \frac{4 \pi^{2}}{9 h_{0}^{2}}$. Throughout our numerical calculations we will work with $u_{k}=1$, which satisfies the first condition easily, while it requires from the second that $h_{0} \ll \frac{2 \pi}{3}$. This condition is also easily satisfied by choosing $u_{0} \gg u_{k}=1^{10}$. For such values of $u_{0}, f(u) \sim 1$ for all $u \geq u_{0}$.

The boundary conditions are imposed using (2.21), (2.22) at a point $u=u_{1}$ which we choose as close to $u_{0}$ as allowed by numerics. Generally we were able to reduce $\left(u_{1}-u_{0}\right)$ down to about 0.1 percent of the value of $u_{0}$. Starting from the values of $T\left(u_{1}\right), T^{\prime}\left(u_{1}\right), h\left(u_{1}\right)$ and $h^{\prime}\left(u_{1}\right)$ obtained from (2.21), (2.22) at $u=u_{1}$, the system was allowed to evolve to larger values of $u$. Figure 2 shows an example for $u_{0}=12.7$. Solutions for both $h(u)$ and $T(u)$ are shown.
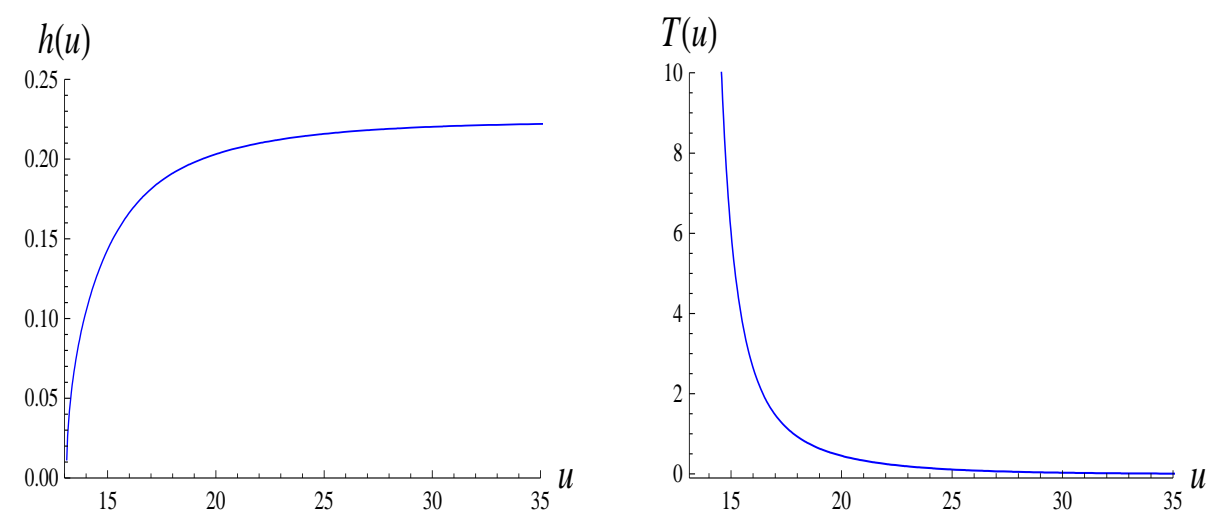

Figure 2: The brane profile and the tachyon solution for $u_{0}=12.7$.

\subsection{Verification of the UV and IR analytic solutions}

From the numerical solutions one can verify that $h(u)$ and $T(u)$ are given by the forms (2.21), (2.22), for $u \sim u_{0}$. Figure 3 shows the impressive fits between the numerical data and the analytical expectations for the powers of $\left(u-u_{0}\right)$ for $h(u)$ and $T(u)$. We have plotted $h(u) / h^{\prime}(u)$ and $T(u) / T^{\prime}(u)$, calculated from the numerical solutions, as functions of $u$. The numerical data are plotted in dashed lines while the theoretical solutions are plotted in solid lines. As one can see, these graphs are linear at the IR end and their slopes turn out to be close to the expected values 0.5 and -2 respectively. In fact, the numerical and the theoretical curves entirely overlap in the IR region of $u$, as shown in Figure 3. At the other end also, namely for large $u$, one can verify that the numerical

\footnotetext{
${ }^{10} \mathrm{As}$ we shall see below, the asymptotic separation decreases with increasing value of $u_{0}$, as is the case for the SS model.
} 

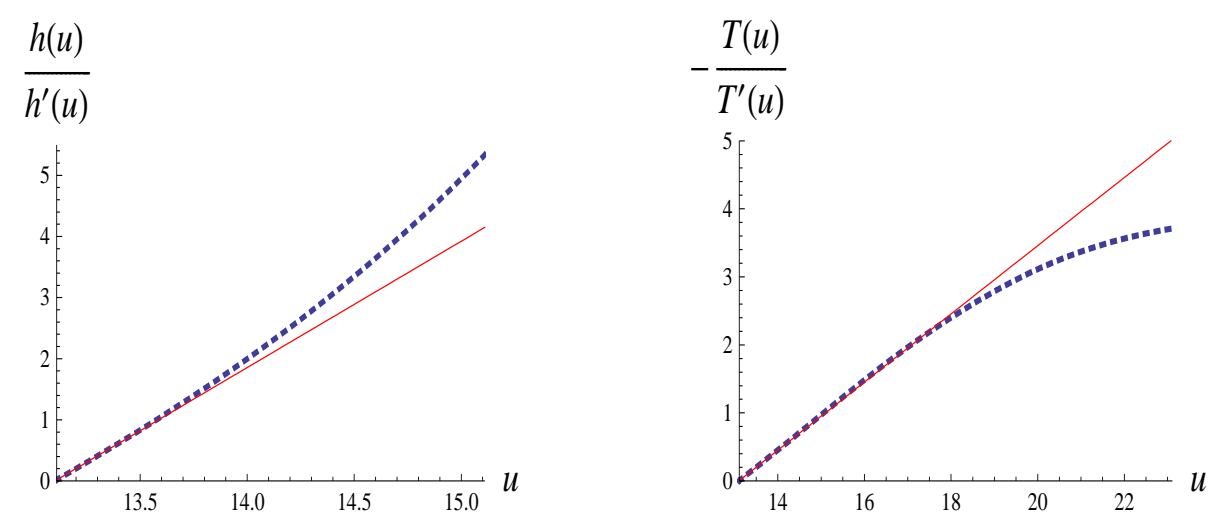

Figure 3: Numerical verification of exponents in the IR behaviour of brane profile and tachyon. The fits give the two exponents respectively to be 0.50 and -2.07 for $u=13.1$.

solutions have the analytic forms (2.18), (2.16). The goodness of the fits of these analytic forms to numerical data is shown in Figure $\$$ where again the two curves overlap in the asymptotic region of $u$. The fits yield values of the four parameters: $h_{0}=0.224, h_{1}=-16068, T_{+}=29194.5, T_{-}=-1.25 \times 10^{-4}$ for $u_{0}=13.1$.
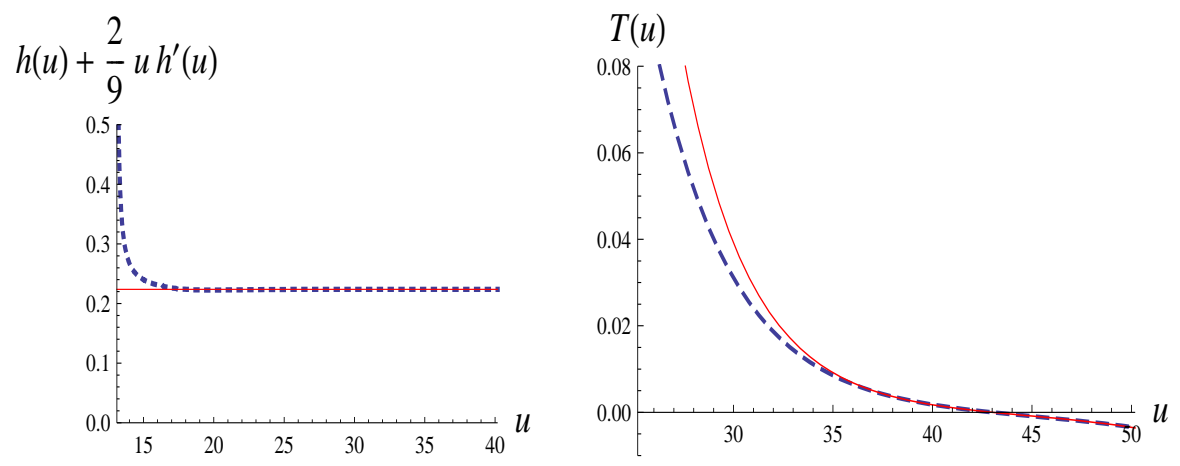

Figure 4: Numerical verification of the asymptotic form of the brane profile and the tachyon.

\subsection{Behaviour of the non-normalizable part}

For $T_{-} \neq 0$, extending numerical calculations much beyond the values of $u$ shown in Figure 2 meets with a difficulty. It turns out that for small $u_{0}, T_{-}$is positive. Since $T_{-}$is the coefficient of the rising exponential in $T(u)$, for a sufficiently large value of $u$ this term dominates and so $T(u)$ begins to rise ${ }^{11}$. Eventually, $T$ becomes so large that the conditions under which the asymptotic solutions (2.18), (2.16) were obtained no longer apply. Figure 5 illustrates this; it shows the solutions for $u_{0}=12.7$ for two different large values of $u$. In Figure 5(a), after falling very fast,

\footnotetext{
${ }^{11}$ We would like to thank Matt Headrick for a discussion on this point and some other aspects of our numerical calculations.
} 


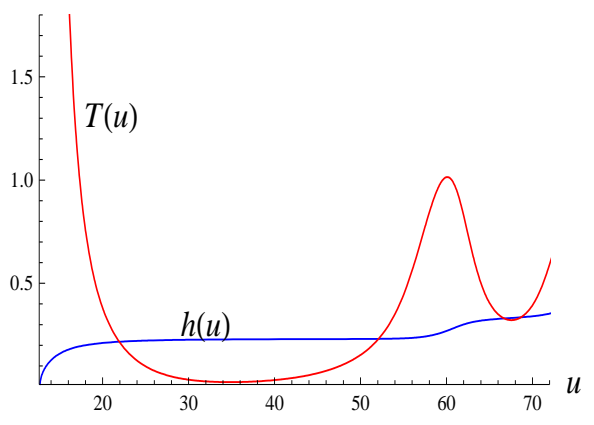

(a)

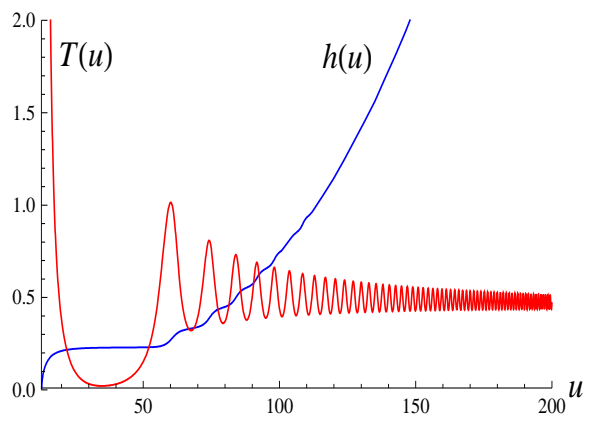

(b)

Figure 5: Solutions for two different large values of $u$.

$T$ rises and then falls again. Almost simultaneous with this is a rapid rise of $h$ from one nearly constant value to a higher constant value. Evidently, this behaviour continues indefinitely with $u$, as can be seen in Figure 5(b) ${ }^{12}$.

The value of $T_{-}$decreases with increasing $u_{0}$. This can be easily deduced from the fact that the maximum value of $u$ up to which the asymptotic solutions (2.16), (2.18) apply, namely before the oscillations begin, increases with increasing $u_{0}$. Figure 6 illustrates this by showing the solutions for increasing values of $u_{0}$, close to where $T_{-}$is small. As one can see, increasing the value of $u_{0}$ by a very small amount, from $u_{0}=13$ to $u_{0}=13.0878$, dramatically increases the threshold for oscillatory behaviour of $T$ from $u \sim 50$ to $u \sim 120$ ! As $u_{0}$ increases further, $T_{-}$decreases,
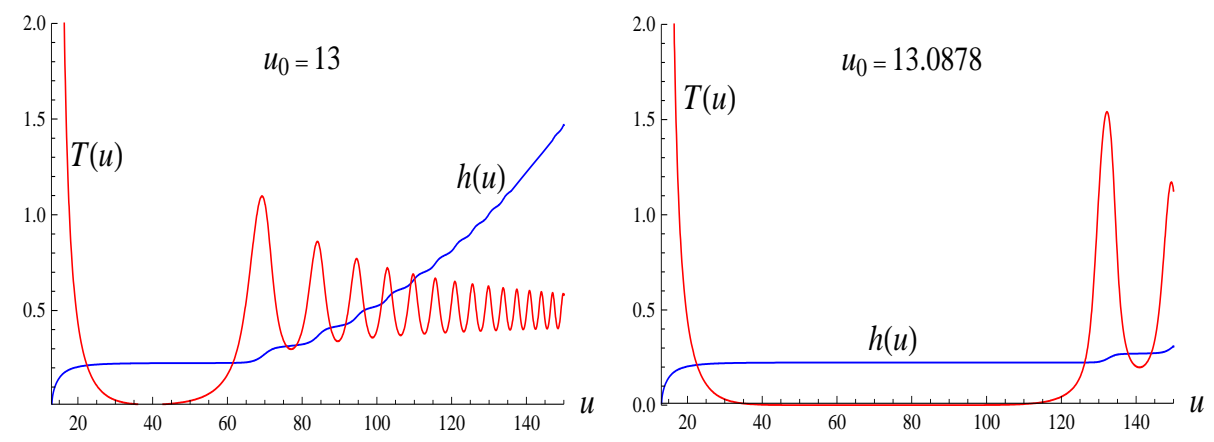

Figure 6: Numerical solutions for increasing values of $u_{0}$ for positive $T_{-}$.

becomes zero ${ }^{13}$ and eventually negative. Since we want to interpret $T_{-}$as the bare

\footnotetext{
${ }^{12}$ In [20], the authors claim that this effect is due to sensitivity of the solutions to the boundary conditions at the infrared end at $u=u_{1}$, which must necessarily be chosen slightly away from the actual value $u_{0}$. We have not found any evidence for this sensitivity. On the other hand, it is clear that the approximation made in deriving the asymptotic solution, (2.16), (2.18), must break down for sufficiently large $u$, for any non-zero value of $T_{-}$. We see convincing numerical evidence for this. Further evidence of this follows.

${ }^{13}$ We have found that $T_{-}=1.92 \times 10^{-9}$ at $u_{0} \sim 13.0877781$. Fine-tuning $u_{0}$ such that $T_{-}$is precisely zero is hard. This requires numerical methods which are beyond the scope of those used here. However, the trend is clear from Figure 6 and Figure 7 .
} 
quark mass parameter, negative values for it are allowed. However, a large value for $\left|T_{-}\right|$will eventually again make $T$ large in magnitude for large enough $u$. So once again we expect that at some sufficiently large $u, T$ will become so large that the conditions under which the asymptotic solutions (2.16), (2.18) were obtained no longer apply. So, as before, one should find oscillations in $T(u)$, which now start at smaller and smaller $u$ as $u_{0}$ grows. This is indeed seen to be the case, as is evident in Figure 7. This happens because $\left|T_{-}\right|$grows with $u_{0}$, beyond the value
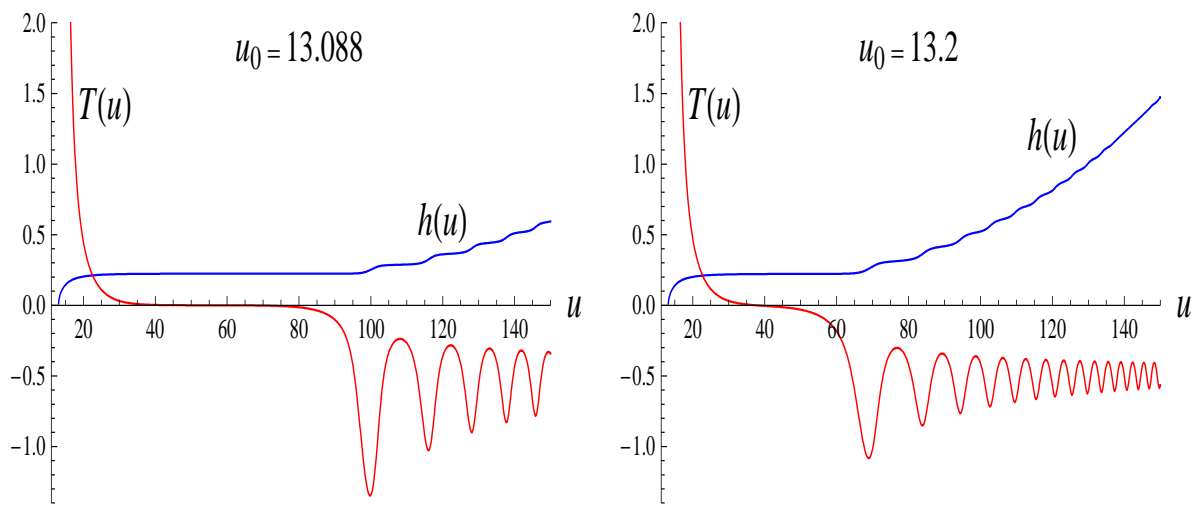

Figure 7: Numerical solutions for increasing values of $u_{0}$ for negative $T_{-}$.

at which it becomes zero. Figure 8 shows the change of $T_{-}$with $u_{0}$. We see that

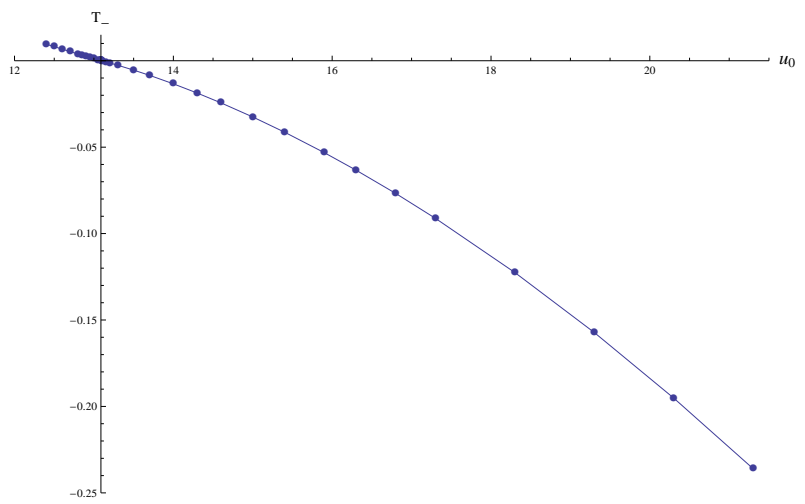

Figure 8: $T_{-}$as a function of $u_{0}$.

$T_{-}$vanishes at $u_{0} \sim 13.0878$ and $\left|T_{-}\right|$grows on both sides away from this value. It is hard to understand what is special about this value of $u_{0}$. One might have thought that the role of zero mass would be played by the antipodal configuration, which has $u_{0}=u_{k}$, and is beyond our approximation. It is possible that this is an artifact of using the approximate action, (2.3), valid for a noncompact $x^{4}$ coordinate, although the value $u_{0} \sim 13.0878$ is fairly large and seems to be within the validity of our approximation. We also note that for negative $T_{-}$, negative $T(u)$ can be avoided by imposing a suitable cut-off on $u$. As we have already discussed, the cut-off is 
in any case required to fulfil the condition (2.19) so that the asymptotic solutions (2.16), (2.18) may apply.

\subsection{Behaviour of the asymptotic brane-antibrane separation}

Another interesting quantity is the asymptotic brane-antibrane separation, $h_{0}$, as a function of $u_{0}$. This quantity has been plotted in Figure 9. We see that $h_{0}$ steadily

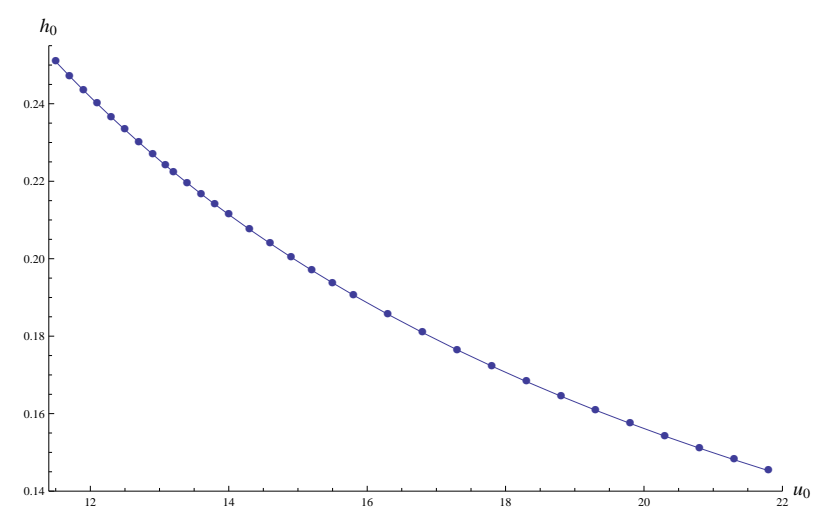

Figure 9: $h_{0}$ as a function of $u_{0}$.

decreases through the special value $u_{0} \sim 13.0878$. Although we do not have an analytical formula for the dependence of $h_{0}$ on $u_{0}$ for large values of the latter, the trend in Figure 9 seems to indicate that it decreases to zero as $u_{0}$ becomes large. Presumably the brane-antibrane pair overlap and disappear as $u_{0}$ goes to infinity. This is consistent with the trend of increasing bare quark mass for increasing values of $u_{0}$ (far beyond $u_{0} \sim 13.0878$ ) which we have seen in Figure 8. Therefore, unlike in the Sakai-Sugimoto model, the disappearance of the brane-antibrane pair for $u_{0}=\infty$ can be understood in the present setup as the infinite bare quark mass limit.

It should be clear from the above discussion that the limit $h_{0} \rightarrow 0$ does not reduce to the case of overlapping $D 8$-branes and $\overline{D 8}$-branes considered in 19 . For this case, one must begin afresh with $x_{i}^{4}=0, l=0$ in the action (2.3). However, the classical equation for $T$ can be obtained from the equation (2.13) by setting $h=0$ in it. As above, we find that solutions which are divergent in the IR depend on only one free parameter. For further details about the tachyon solutions in this case, we refer the interested reader to the Appendix A.

\subsection{Comparison with the Sakai-Sugimoto solution}

Finally, we must ensure that the solution with the tachyon has lower energy compared to the SS model. The energy density in the modified model is given by

$$
E_{\mathrm{T}}=2 V_{4} R^{9} V(0) \int_{u_{0}}^{u_{\max }} d u E_{\mathrm{T}}(u)
$$




$$
E_{\mathrm{T}}(u)=u^{13 / 4} \frac{V(T)}{V(0)} \sqrt{u^{-3 / 2}+\frac{1}{4} u^{3 / 2} h^{\prime}(u)^{2}+T^{\prime}(u)^{2}+T(u)^{2} h(u)^{2}},
$$

while for the SS model it is given by

$$
\begin{aligned}
E_{\mathrm{SS}} & =2 V_{4} R^{9} V(0) \int_{u_{0}}^{u_{\max }} d u E_{\mathrm{SS}}(u), \\
E_{\mathrm{SS}}(u) & =u^{13 / 4} \sqrt{u^{-3 / 2}+\frac{1}{4} u^{3 / 2} h_{\mathrm{SS}}^{\prime}(u)^{2}} .
\end{aligned}
$$

To get these expressions for energy density, we have set $f(u)$ to unity, which is a good approximation for large $u_{0}$. Also, in the SS model one must use the solution of the tachyon free equation, $h_{\mathrm{SS}}^{\prime}(u)=2 u_{0}^{4} u^{-3 / 2}\left(u^{8}-u_{0}^{8}\right)^{-1 / 2}$.

Close to $u_{0}$, in the IR, the exponentially vanishing tachyon potential suppresses contribution to $E_{\mathrm{T}}$ compared to $E_{\mathrm{SS}}$. Since the UV solutions for the two models are almost identical ${ }^{14}$, one might argue that the energy for the modified model must be lower than that for the SS model. However, for $u \gtrsim u_{0}$ there is a competition between the exponentially vanishing tachyon potential and the power law increase of the square-root factor coming from $\left|T^{\prime}\right|$ in the integrand $E_{\mathrm{T}}(u)$ in (3.1). This results in a local maximum in $E_{\mathrm{T}}(u)$ at some value of $u$, which can be easily estimated analytically. The relevant quantity,

$$
e^{-\frac{\pi}{4} u_{0}^{3 / 2}\left(u-u_{0}\right)^{-2}}\left(u-u_{0}\right)^{-3}
$$

has a maximum at $u=u_{0}+\left(\frac{\pi}{6}\right)^{1 / 2} u_{0}^{3 / 4}$. For small $u_{0}$, the position of the maximum is close to $u_{0}$, so in this case the argument about the IR behaviour of the integrand in (3.1) is not very clean, except in the very deep IR. But since the position of the maximum grows with increasing $u_{0}$ as $u_{0}^{3 / 4}$, our argument should hold for large values of $u_{0}$, which is precisely where the action for the modified model can be trusted. However, the expression used for estimating the position of the local maximum breaks down if it is too far away from $u_{0}$. So, in practice we need to do a numerical calculation to see what the real story is. As we will see in the numerical plots given below, what really happens is that for relatively large values of $u_{0}$ the integrand $E_{\mathrm{T}}(u)$ increases rapidly at first, then slows down almost to a constant and finally settles into an asymptotic power law increase similar to that of the integrand $E_{\mathrm{SS}}(u)$ for the SS model. Moreover, the place where the rapid increase begins shifts to larger values of $u$ as $u_{0}$ increases, in accordance with the above expectation.

We have numerically evaluated the integrals in (3.1) and (3.2). Because the relation between $u_{0}$ and the asymptotic brane-antibrane separation is different in

\footnotetext{
${ }^{14}$ There is a caveat here. Strictly speaking this is true only when the coefficient of the nonnormalizable term, $T_{-}$, in the asymptotic tachyon solution (2.16) vanishes. As we have discussed, when $T_{-}$is nonzero, one must introduce a cut-off, $u_{\max }$, chosen carefully such that the asymptotic solution is satisfied. In particular, one must ensure $T$ is positive in the region below $u_{\max }$. In the calculations reported here and earlier in this section, this is what we have done.
} 
the two models, a given value of $u_{0}$ corresponds to two different values of the latter and vice versa. We have chosen to do the comparison for the same value of the asymptotic brane-antibrane separation in the two models, but the conclusions are similar with the other choice as well. In Figure 10 we have plotted numerical
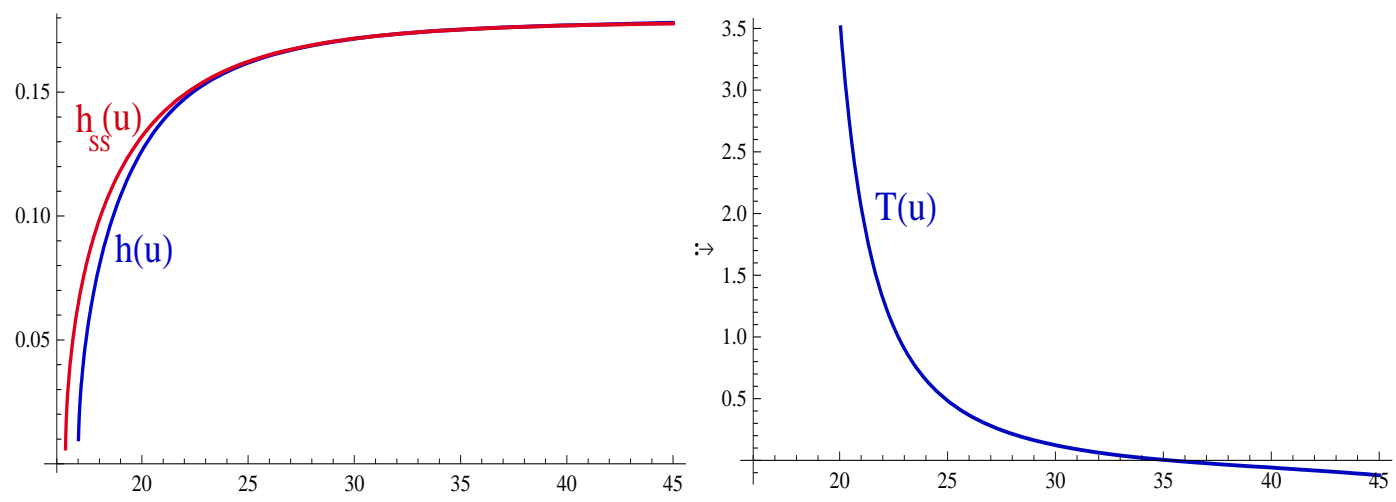

Figure 10: $h(u)$ and $T(u)$ profiles for $u_{0}=17$. For comparison, $h_{\mathrm{SS}}$ profile has also been plotted after adjusting the value of $u_{0}$ to 16.4 for it since this value of $u_{0}$ produces the same asymptotic brane-antibrane separation.

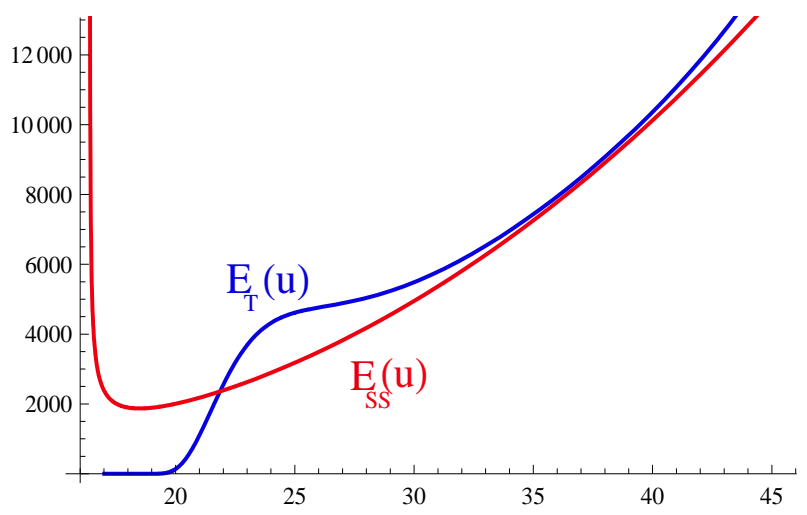

Figure 11: The energy density integrands $E_{\mathrm{SS}}(u)$ and $E_{\mathrm{T}}(u)$. The rapid rise of the latter in the IR is clearly seen. The divergence between the two curves in the asymptotic region, $u \gtrsim u_{\max }$, is due to a nonzero $T_{-}$.

solutions for $h(u)$ and $T(u)$ for $u_{0}=17^{15}$. For comparison with the SS model, we have also plotted $h_{\mathrm{SS}}$ after adjusting the value of $u_{0}$ for it to produce the same value of the asymptotic brane-antibrane separation. The required value turns out to be $u_{0}=16.4$. The corresponding energy density integrands, $E_{\mathrm{T}}(u)$ and $E_{\mathrm{SS}}(u)$, have been plotted in Figure 11. We can clearly see the rapid rise of $E(u)$ in the IR, the subsequent flattening out and finally the power-law rise in

\footnotetext{
${ }^{15}$ Similar behaviour is seen for values of $u_{0} \gtrsim 14$. Below $u_{0} \sim 14$, however, the energy difference becomes very small and even reverses sign. This may be connected with the breakdown of the approximate action in this region, similar to the observation of a zero quark mass at $u_{0} \sim 13.01$.
} 
the asymptotic region. Using $u_{\max }=35.32{ }^{16}$, numerical evaluation of the integrals gives $\left(E_{\mathrm{T}}-E_{\mathrm{SS}}\right)=-300.3$. Therefore, the solution with the tachyon taken into account corresponds to a lower energy state.

\section{The chiral condensate}

By the standard dictionary of AdS/CFT [25, 22, 23, 24], once we have identified $T_{-}$ with the quark mass parameter, we should identify $T_{+}$with the chiral condensate. However, it is not clear that the standard rules apply to the present case of a boundary theory which is not a CFT and has a scale. Moreover, the fact that there is no known lift of $D 8$-brane to 11-dimensions forces an essential cut-off in the theory with flavours. In fact, for a non-zero value of $T_{-}$, the real cut-off is much lower, as we have seen from numerical computations in the last section. Despite these difficulties, we will assume that the identification of sources in the boundary theory with boundary values of bulk fields holds in the theory with cut-off.

There is an additional difficulty in the present case. As we have seen above, the desired solutions have only one independent parameter, which we take to be $T_{-}$. The other three parameters, $T_{+}, h_{0}$ and $h_{1}$ should then be considered to be functions of $T_{-}$. Thus, the chiral condensate cannot be computed naively by varying the on-shell flavour brane action w.r.t. $T_{-}$, since this would also include contributions from the variation of the other three parameters with $T_{-}$. The one-parameter solutions that we have found constitute the most general class of space-time independent solutions with the specified boundary conditions ${ }^{17}$. Therefore, if we only want to make a variation of $T_{-}$only, we must go out of the present one-parameter class of solutions to more general solutions, which are space-time dependent, in addition to being dependent on $u$, and have enough parameters. These solutions to $(u, x)$-dependent equations should have the same singularities at $u=u_{0}$ as the solutions in (2.21) and (2.22). Moreover, the asymptotic solutions should have the form of (2.16) and (2.18) with $x$-dependent coefficients. If solutions satisfying these conditions exist and have enough parameters, then we can make the required variation of $T_{-}$only and identify $T_{+}$as the condensate in a coherent state formed from fluctuations of $T$ and $h$ (scalar mesons) around the ground state with broken chiral symmetry. Specializing to the $x$-independent case, after varying the on-shell action, then, gives us the condensate in the vacuum state. What we, therefore, need to do is to analyse the $x$-dependent case to see if the required solutions exist. This is what we will do next.

\footnotetext{
${ }^{16}$ This is the value at which $T(u)$ vanishes. The asymptotic form, (2.16), fits the numerically computed $T(u)$ in the range $33 \leq u \leq u_{\max }$ to better than a percent with the parameter values $h_{0}=0.179, T_{+}=28904, T_{-}=-0.0937$.

${ }^{17}$ These boundary conditions are (i) vanishing tachyon and fixed brane-antibrane separation asymptotically and (ii) divergent tachyon and vanishing brane-antibrane separation at some point in the bulk.
} 


\subsection{Action for $(u, x)$-dependent $T$ and $h$}

The full $(u, x)$-dependent action for tachyon and brane-antibrane separation is given by

$$
S=-\frac{2 V_{4}}{R^{9}} \int d^{4} x \int d u u^{13 / 4} V(T) \sqrt{d_{T}} \sqrt{\operatorname{det}(1+K)},
$$

where $K$ is the matrix with the elements

$$
\begin{aligned}
K_{\nu}^{\mu} & =\frac{f}{4 Q} \partial^{\mu} h \partial_{\nu} h+\frac{u^{-3 / 2}}{Q} \partial^{\mu} T \partial_{\nu} T, \\
K^{\mu}{ }_{u} & =\frac{f}{4 Q} h^{\prime} \partial^{\mu} h+\frac{u^{-3 / 2}}{Q} T^{\prime} \partial^{\mu} T, \\
K^{u} & =\frac{f u^{3 / 2}}{4 d_{T}} h^{\prime} \partial_{\mu} h+\frac{1}{d_{T}} T^{\prime} \partial_{\mu} T, \\
K_{u}^{u} & =0 .
\end{aligned}
$$

To look for a generalization of the $x$-independent solutions for equations of motion derived from this action, the most obvious thing to do is to generalize the earlier solutions by making all parameters functions of $x$. In particular, this means making $u_{0}$, the place where the flavour brane and antibrane meet, a function of $x$. For $u \sim u_{0}$, expansion of this solution around a constant $u_{0}$ is singular, since it involves arbitrary higher powers of $1 /\left(u-u_{0}\right)$. Therefore, we do not expect analysis of (4.1) by expanding in small fluctuations around the $x$-independent solution to work for $u$ close to $u_{0}$. This is confirmed by explicit fluctuation calculations in Appendix $\mathrm{B}$. We need to go beyond small fluctuations analysis of (4.1) and this requires us to get an exact expression for the determinant in terms of space-time derivatives of $T$ and $h$.

A direct calculation of $\operatorname{det}(1+K)$ is tedious, but the calculation can be simplified using a trick which has been described in Appendix Q, where a rather simple expression for the determinant has been obtained. The complete 5-dimensional action then reads

$$
S=-\frac{2 V_{4}}{R^{9}} \int d^{4} x \int d u u^{13 / 4} V(T) \sqrt{\Delta_{T}}
$$

where $\Delta_{T}=d_{T} \Delta$ and we have defined

$$
\Delta \equiv 1+\beta_{1}(\partial T)^{2}+\beta_{2}(\partial h)^{2}+2 \beta_{3}(\partial h . \partial T)+\beta_{4}\left[(\partial T)^{2}(\partial h)^{2}-(\partial h . \partial T)^{2}\right] .
$$

The $\beta$ 's are given by

$$
\beta_{1}=\frac{u^{-3 / 2}}{Q}\left(1-\frac{T^{\prime 2}}{d_{T}}\right), \quad \beta_{2}=\frac{f}{4 Q}\left(1-\frac{f u^{3 / 2} h^{\prime 2}}{4 d_{T}}\right), \quad \beta_{3}=-\frac{f h^{\prime} T^{\prime}}{4 Q d_{T}}, \quad \beta_{4}=\beta_{1} \beta_{2}-\beta_{3}^{2} .
$$

As a check on the action (4.3), we note that it reduces to the action (2.10) if $T$ and $h$

are $x$-independent. Also, it correctly reproduces the action (B.1) which only retains 
terms that are quadratic in space-time derivatives of $T$ and $h$. This latter action was derived independently by expanding $\operatorname{det}(1+K)$ in powers of $K$ and retaining only the first nontrivial correction.

The equations of motion that follow from the action (4.3) are rather complicated and have been derived in Appendix Q, (C.13) and (C.14). As we did in the $x$ independent case, we will solve these equations in the two limiting cases of large $u$ and $u \sim u_{0}$.

$u \rightarrow u_{\max }$ : In this limit, $h(u, x)$ goes to a fixed value $h_{0}(x)$, which is assumed to be a slowly varying function of $x$. We will also assume that $T$ and all its derivatives are small in this limit. Then the equations (C.13) and (C.14) can be approximated as

$$
\begin{array}{r}
-\left(u^{4} T^{\prime}(u, x)\right)^{\prime}+\left(h_{0}(x)\right)^{2} u^{4} T(u, x)=0 \\
\left(u^{\frac{11}{2}} h^{\prime}(u, x)\right)^{\prime}=0 .
\end{array}
$$

The space-time derivatives are comparatively suppressed by powers of $1 / u$ and hence have been ignored. These equations are identical to (2.15) and (2.17) and so have solutions similar to (2.16) and (2.18), but now with parameters that are functions of $x$ :

$$
\begin{aligned}
T(u, x) & =\frac{1}{u^{2}}\left(T_{+}(x) e^{-h_{0}(x) u}+T_{-}(x) e^{h_{0}(x) u}\right), \\
h(u, x) & =h_{0}(x)-h_{1}(x) u^{-9 / 2} .
\end{aligned}
$$

$u \rightarrow u_{0}$ : The analysis in this limit is somewhat more involved. We assume an ansatz similar to the solutions (2.21) and (2.22), but now with $x$-dependent $u_{0}$ and coefficients:

$$
\begin{aligned}
& h(u, x)=\rho_{0}(x)\left(u-u_{0}(x)\right)^{1 / 2}+\rho_{1}(x)\left(u-u_{0}(x)\right)^{3 / 2}+\cdots, \\
& T(u, x)=\sigma_{0}(x)\left(u-u_{0}(x)\right)^{-2}+\sigma_{1}(x)\left(u-u_{0}(x)\right)^{-1}+\cdots
\end{aligned}
$$

As consequence of this ansatz, one can show that

$$
\begin{aligned}
& \partial_{\mu} h=-h^{\prime}\left[\partial_{\mu} u_{0}-\frac{2 \partial_{\mu} \rho_{0}}{\rho_{0}}\left(u-u_{0}\right)+\cdots\right], \\
& \partial_{\mu} T=-T^{\prime}\left[\partial_{\mu} u_{0}+\frac{\partial_{\mu} \sigma_{0}}{2 \sigma_{0}}\left(u-u_{0}\right)+\cdots\right] .
\end{aligned}
$$

These relations are correct to the order shown. Putting all this in the equation of motion for $T$, C.13), we see that this equation is satisfied to the leading order provided the following condition holds:

$$
\frac{13}{4 u_{0}}-\frac{\sqrt{\pi}}{2} \sigma_{0} \rho_{0}^{2}=u_{0}^{-3 / 2} \partial_{\mu}\left(u_{0}^{-3 / 2} \partial^{\mu} u_{0}\right)-\frac{1}{2} u_{0}^{-3} \partial^{\mu} u_{0} \frac{\partial_{\mu}\left(u_{0}^{-3}\left(\partial u_{0}\right)^{2}\right)}{1+u_{0}^{-3}\left(\partial u_{0}\right)^{2}} .
$$


In obtaining this we have set $f_{0}=1$. Similarly, from (C.14) one gets the condition

$$
\sigma_{0}=\frac{\sqrt{\pi}}{4}\left(u_{0}^{3 / 2}+u_{0}^{-3 / 2}\left(\partial u_{0}\right)^{2}\right) .
$$

If $u_{0}$ is a constant independent of $x$, then from equations (4.12) and (4.13) one gets

$$
\sigma_{0}=\frac{\sqrt{\pi}}{4} u_{0}^{3 / 2}, \quad \rho_{0}=\sqrt{\frac{26}{\pi u_{0}}} u_{0}^{-3 / 4} .
$$

These reproduce the $x$-independent solutions in (2.21) and (2.22), remembering that we have set $f_{0}=1$. Let us now consider a small fluctuation around this constant solution. Linearizing the equations (4.12) and (4.13) in fluctuations, we get

$$
\delta \sigma_{0}(x)=\frac{3 \sqrt{\pi}}{8} u_{0}^{1 / 2} \delta u_{0}(x), \quad \delta \rho_{0}(x)=-\frac{4 u_{0}^{-13 / 4}}{\sqrt{26 \pi}}\left(\partial^{2}+\frac{65}{8} u_{0}\right) \delta u_{0} .
$$

Now, clearly we could choose the fluctuation $\delta u_{0}(x)$ to be such that $\delta \rho_{0}(x)$ vanishes. Under such an infinitesimal change of $u_{0}, \sigma_{0}$ would change, but not $\rho_{0}$. It is this kind of greater freedom in independently varying the parameters of the solution that we have wanted. Presumably in higher orders the situation gets better because there are more terms in the ansatz (4.9) and for each coefficient there is some freedom because of the space-time dependence. It would be nice to analyse the higher order terms, but that is beyond the scope of this work. Here we will assume that the introduction of space-time dependence as above can give us the required freedom to do the calculation of the condensate as follows.

Finally, let us compare the solution (4.14), (4.15) with the solution obtained by the singular perturbation expansion in Appendix B, (B.31). Expanding (4.9) around constant $u_{0}$ solution to the lowest nontrivial order in $\epsilon \equiv\left(u-u_{0}\right)$ and comparing with (B.28), we get the relations

$$
\begin{aligned}
\varphi_{0}(x) & =2 \delta u_{0}(x), & \varphi_{1}(x) & =\frac{1}{\sigma_{0}}\left(\delta \sigma_{0}(x)+\sigma_{1} \delta u_{0}(x)\right), \\
\vartheta_{0}(x) & =-\frac{1}{2} \delta u_{0}(x), & \vartheta_{1}(x) & =\frac{1}{\rho_{0}}\left(\delta \rho_{0}(x)-\frac{3}{2} \rho_{1} \delta u_{0}(x)\right) .
\end{aligned}
$$

These relations involve not only the leading order parameters (4.14) of the constant solution, but also the nonleading parameters $\sigma_{1}, \rho_{1}$, which are given by

$$
\sigma_{1}=\frac{\sigma_{0}}{6 u_{0}}, \quad \rho_{1}=-\frac{5 \rho_{0}}{8 u_{0}} .
$$

Using (4.14)-(4.17), one can show that the equations in (B.31) are satisfied. This equivalence is, however, only formal. As we have argued above, the method given in this section is the correct one to use since it does not involve a singular expansion in arbitrarily high powers of $1 /\left(u-u_{0}\right)$. 


\subsection{Condensate in terms of the tachyon solution}

To derive an expression for the condensate, we calculate the variation of the action in (4.3) under a general variation of $T$ and use the equation of motion (C.13) to reduce it to a boundary term:

$$
\delta S=-\left.\frac{2 V_{4}}{R^{9}} \int d^{4} x \frac{V(T) u^{13 / 4}}{\sqrt{d_{T}}} T^{\prime}(u, x) \delta T(u, x)\right|_{u=u_{\max }} .
$$

We have ignored terms with space-time derivatives because from now on we will be specializing to the $x$-independent case, except in the variation $\delta T$, so these terms will drop out. Only the UV boundary contributes to the on-shell action; there is no IR contribution because the tachyon potential vanishes exponentially for the diverging tachyon in the IR. We are only interested in retaining the variation $\delta T_{-}(x)$, so we set $\delta T_{+}(x)$ to zero. Doing this and using (4.8) in (4.18), we get the leading contribution for large $u_{\max }$,

$$
\delta S \approx \frac{2 h_{0} V_{4} V(0)}{R^{9}}\left(T_{+}-T_{-} e^{2 h_{0} u_{\max }}\right) \int d^{4} x \delta T_{-}(x)
$$

On-shell brane actions have UV divergences which need to be removed by the holographic renormalization procedure ${ }^{18}$ to get finite answers for physical quantities. One adds boundary counter terms to the brane action to remove the divergences, following a procedure described in [43]. Our on-shell action (4.19) diverges as the cut-off is removed. This is because, as discussed in section 2.4, we are keeping $T_{+}$and $T_{-} e^{h_{0} u_{\max }}$ fixed as the cut-off is removed and the last term in (4.19) diverges as $e^{h_{0} u_{\max }}$ in this limit. The holographic renormalization procedure has been developed for examples with CFT boundary theories. Since, with the D8-branes present, there is no 11-dimensional description available to us, it is not clear that the procedure described in [43] is applicable to the present case. We will proceed on the assumption that this is the case. Therefore, to subtract the UV divergent term in (4.19), we will add the following counter term to the boundary action,

$$
S_{\mathrm{ct}}=\left.\frac{V_{4} V(0)}{R^{9}} \int d^{4} x \sqrt{-\gamma} h(u, x) T^{2}(u, x)\right|_{u_{\max }},
$$

where $\gamma=-u_{\max }^{8}$ is the determinant of the metric on the 8-dimensional boundary orthogonal to the slice at $u=u_{\max }$. Note that the counter terms must be even in powers of the tachyon because of gauge symmetry. Using the solution (4.8) and retaining only the parameter $T_{-}(x)$, we find that the variation of the counter term action is

$$
\delta S_{\mathrm{ct}}=\frac{2 h_{0} V_{4} V(0)}{R^{9}}\left(T_{+}+T_{-} e^{2 h_{0} u_{\max }}\right) \int d^{4} x \delta T_{-}(x) .
$$

\footnotetext{
${ }^{18}$ For reviews, see 41, 42].
} 
Adding to (4.19), the divergent term drops out and we get the variation of the renormalized action

$$
\delta S_{\text {renorm }} \approx \frac{4 h_{0} V_{4} V(0)}{R^{9}} T_{+} \int d^{4} x \delta T_{-}(x)
$$

Note that the variation of the renormalized action is twice as large as it would have been if we had simply dropped the divergent term ${ }^{19}$ in (4.19).

We are now ready to calculate an expression for the chiral condensate in terms of the parameters of the tachyon solution. The parameters $T_{ \pm}$are dimensionless. To construct a parameter of dimension mass from $T_{-}$, we introduce a scale $\mu$ and define $m_{q}=\mu\left|T_{-}\right|$. Then, identifying the chiral condensate $\chi \equiv<\overline{q_{L}} q_{R}>$, with $\delta S_{\text {renorm }} / \mu \delta T_{-}(x)$, we get

$$
\chi \approx \frac{4 h_{0} V_{4} V(0)}{\mu R^{9}} T_{+}
$$

We see that the parameter $T_{+}$determines the condensate. Figure 12 shows a plot of $T_{+}$as a function of $T_{-}$for $T_{-} \sim 0$. $T_{+}$seems to attain a maximum value at $T_{-}=0$

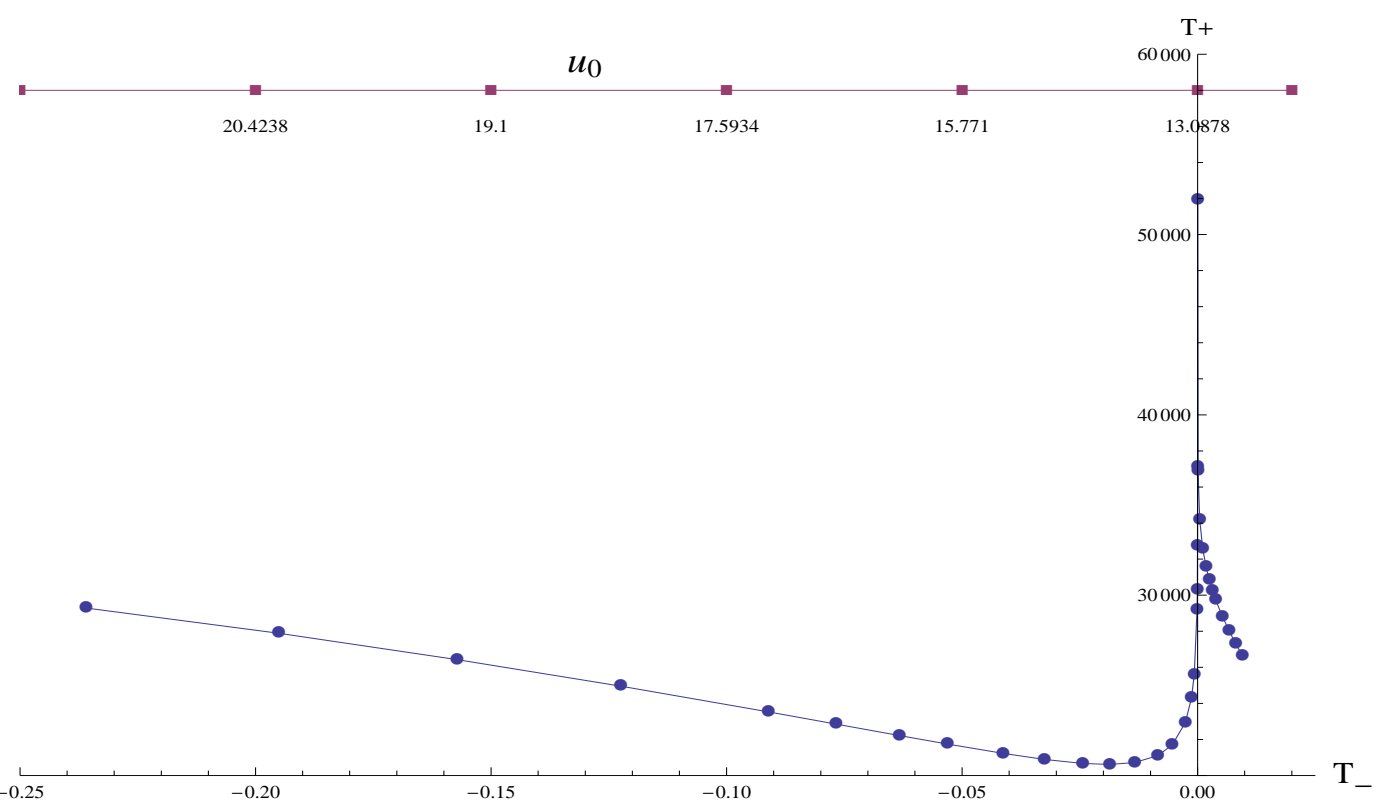

Figure 12: $T_{+}$as a function of $T_{-}$.

and drops off rapidly, at least for small values of $\left|T_{-}\right|$.

\footnotetext{
${ }^{19}$ In (4.19), it is inconsistent to drop the term proportional to $T_{-}$in the limit of large cut-off, holding $T_{+}$and $T_{-} e^{h_{0} u_{\max }}$ fixed. In fact, it is the $T_{-}$term that dominates in the action $(4.19)$ in this limit. Taking a different limit that allows one to simply drop this term creates difficulties in the calculation of the pion mass, see section 5.3. Consistency with the chiral condensate calculation then demands that the term proportional to $T_{+} T_{-}$be dropped in the pion mass calculation since it is smaller than the $T_{+}^{2}$ term.
} 


\section{The meson spectra}

In this section we will discuss the spectra for various low spin mesons which are described by the fluctuations of the flavour branes around the classical solution 20. The action for the fluctuations of the gauge fields can be computed from (2.3). Parametrizing the complex tachyon $\tau$ in terms of its magnitude and phase, $\tau=T e^{i \theta}$, we get the following action, correct to second order in the fluctuations:

$$
\begin{aligned}
\Delta S_{\text {gauge }}= & -\int d^{4} x d u\left[a(u) A_{u}^{2}+b(u) A_{\mu}^{2}+c(u)\left(\left(F_{\mu \nu}^{V}\right)^{2}+\left(F_{\mu \nu}^{A}\right)^{2}\right)+e(u) F_{\mu u}^{A} A^{\mu}\right. \\
& \left.+d(u)\left(\left(F_{\mu u}^{V}\right)^{2}+\left(F_{\mu u}^{A}\right)^{2}\right)\right] \\
a(u)= & R^{-15} V_{4} V(T) u^{13 / 4} \frac{T^{2}}{\sqrt{d_{T}}} \\
b(u)= & R^{-3} V_{4} V(T) u^{7 / 4} \sqrt{d_{T}} \frac{T^{2}}{Q}\left(1+\frac{f^{2} T^{2} h^{2} h^{2}}{4 d_{T}} u^{3}\right) \\
c(u)= & \frac{R^{3}}{8} V_{4} V(T) u^{1 / 4} \sqrt{d_{T}} \\
d(u)= & R^{-9} V_{4} V(T) u^{7 / 4} \frac{Q}{4 \sqrt{d_{T}}} \\
e(u)= & R^{-6} V_{4} V(T) u^{13 / 4} \frac{f T^{2} h h^{\prime}}{2 \sqrt{d_{T}}}
\end{aligned}
$$

Here $F_{\mu \nu}^{V}$ is the usual field strength for the vector gauge field $V=\left(A_{1}+A_{2}\right)$ and $F_{\mu \nu}^{A}$ is the field strength for the gauge-invariant combination of the axial vector field and the phase of the tachyon, $A=\left(A_{1}-A_{2}-\partial \theta\right)$. However,

$$
F_{\mu u}^{V}=-F_{u \mu}^{V}=\partial_{\mu} V_{u}-R^{3} \partial_{u} V_{\mu}, \quad F_{\mu u}^{A}=-F_{u \mu}^{A}=\partial_{\mu} A_{u}-R^{3} \partial_{u} A_{\mu} .
$$

The relative factor of $R^{3}$ simply reflects the change of variables (2.9)).

The gauge field $V_{\mu}(x, u)$ gives rise to a tower of vector mesons while the fields $A_{\mu}(x, u)$ and $A_{u}(x, u)$, which are gauge invariant, give rise to towers of axial and pseudoscalar mesons. Notice that the coefficients $a(u), b(u)$ and $e(u)$ vanish if the tachyon is set to zero. In the absence of the tachyon, the vector and axial vector mesons acquire masses because of a nonzero $d(u)$, but there is always a massless "pion" ${ }^{21}$. The presence of the tachyon is thus essential to give a mass to the "pion". Also note that with the tachyon present, the masses of the vector and axial vector mesons are in principle different.

\footnotetext{
${ }^{20}$ For a general review of mesons in gauge/gravity duals, see 44.

${ }^{21}$ Strictly speaking, for the $U(1)$ case under discussion, this pseudoscalar is the $\eta^{\prime}$. It is massless here because of the $N_{c} \rightarrow \infty$ limit in which we are working.
} 


\subsection{Vector mesons}

We will be using the gauge $V_{u}=0$. Expanding in modes, we have

$$
V_{\mu}(x, u)=\sum_{m} V_{\mu}^{(m)}(x) W_{m}(u),
$$

where $\left\{W_{m}(u)\right\}$ form a complete sets of basis functions. These satisfy orthonormality conditions which will be determined presently. The fields $\left\{V_{\mu}^{(m)}(x)\right\}$ form a tower of vector mesons in the physical $(3+1)$-dimensional space-time. In terms of these fields, the vector part of the action (5.1) takes the form,

$$
\Delta S_{\text {gauge }}^{V}=-\int d^{4} x \sum_{m, n}\left[Q_{m n}^{V} F_{\mu \nu}^{V(m)} F^{V(n) \mu \nu}+L_{m n}^{V} V_{\mu}^{(m)} V^{(n) \mu}\right],
$$

where $F_{\mu \nu}^{V(m)}$ are the usual $(3+1)$-dimensional $U(1)$-invariant field strengths for the vector potentials $\left\{V_{\mu}^{(m)}\right\}$. Also, we have defined

$$
Q_{m n}^{V}=\int d u c(u) W_{m}(u) W_{n}(u), \quad L_{m n}^{V}=R^{6} \int d u d(u) W_{m}^{\prime}(u) W_{n}^{\prime}(u) .
$$

In addition, we choose the basis functions $\left\{W_{m}(u)\right\}$ to satisfy the eigenvalue equations

$$
-R^{6}\left(d(u) W_{m}^{\prime}(u)\right)^{\prime}=2 \lambda_{m}^{V} c(u) W_{m},
$$

Using these we see that

$$
L_{m n}^{V}=\frac{1}{2}\left[R^{6}\left(d(u) W_{m}^{\prime}(u) W_{n}(u)\right)_{\partial u}+2 \lambda_{m}^{V} Q_{m n}^{V}\right]+m \leftrightarrow n,
$$

where, as in the previous section, $\partial u$ refers to boundaries in the $u$-direction.

Note that a potential zero mode in the vector sector ${ }^{22}$ can be gauged away using the residual symmetry of making $u$-independent gauge transformations, which is still available after fixing the gauge $V_{u}=0$. This is because a zero mode in this sector can only have a single scalar degree of freedom. This follows from the requirement of finiteness of the the action, (5.9), which cannot be satisfied since the coefficient of the field strength term blows up for a zero mode. Hence its field strength must vanish, leaving behind only a longitudinal degree of freedom.

For the nonzero modes we may, without loss of generality, choose

$$
Q_{m n}^{V}=\frac{1}{4} \delta_{m n}
$$

which, on using (5.12), gives

$$
L_{m n}^{V}=\frac{1}{2} \lambda_{m}^{V} \delta_{m n}
$$

Using (5.13) and (5.14) in (5.9), we get

$$
\Delta S_{\text {gauge }}^{V}=-\int d^{4} x \sum_{m}\left[\frac{1}{4} F_{\mu \nu}^{V(m)} F^{V(m) \mu \nu}+\frac{1}{2} \lambda_{m}^{V} V_{\mu}^{(m)} V^{(m) \mu}\right] .
$$

\footnotetext{
${ }^{22} \mathrm{~A}$ zero mode is defined as a mode which has zero eigenvalue and goes to a constant at infinity.
} 


\subsection{Axial vector and pseudoscalar mesons}

As we have already noted, $A_{\mu}$ and $A_{u}$ are gauge invariant. Expanding in modes, we have

$$
A_{\mu}(x, u)=\sum_{m} A_{\mu}^{(m)}(x) P_{m}(u), \quad A_{u}(x, u)=\sum_{m} \phi^{(m)}(x) S_{m}(u)
$$

where $\left\{P_{m}(u)\right\}$ and $\left\{S_{m}(u)\right\}$ form complete sets of basis functions. These satisfy orthonormality conditions which will be determined presently. The fields $\left\{A_{\mu}^{(m)}(x)\right\}$ and $\left\{\phi^{(m)}(x)\right\}$ form towers of axial vector and pseudoscalar mesons in the physical $(3+1)$-dimensional space-time. In terms of these fields, the axial-vector and pseudoscalar part of the action (5.1) takes the form,

$$
\begin{aligned}
\Delta S_{\text {gauge }}^{A}= & -\int d^{4} x \sum_{m, n}\left[\frac{1}{2} \delta_{m n} \lambda_{m}^{\phi} \phi^{(m)} \phi^{(n)}+Q_{m n}^{A} F_{\mu \nu}^{A(m)} F^{A(n) \mu \nu}+L_{m n}^{A} A_{\mu}^{(m)} A^{(n) \mu}\right. \\
& \left.+K_{m n} \partial_{\mu} \phi^{(m)} \partial^{\mu} \phi^{(n)}+J_{m n} A^{(m) \mu} \partial_{\mu} \phi^{(n)}\right]
\end{aligned}
$$

where $F_{\mu \nu}^{A(m)}$ are the usual $(3+1)$-dimensional $U(1)$-invariant field strengths for the axial vector potentials $\left\{A_{\mu}^{(m)}\right\}$. Also, we have defined

$$
\begin{aligned}
Q_{m n}^{A} & =\int d u c(u) P_{m}(u) P_{n}(u), \\
L_{m n}^{A} & =\int d u\left(R^{6} d(u) P_{m}^{\prime}(u) P_{n}^{\prime}(u)+\left(b(u)+\frac{1}{2} R^{3} e^{\prime}(u)\right) P_{m}(u) P_{n}(u)\right), \\
J_{m n} & =\int d u\left(e(u) P_{m}(u)-2 R^{3} d(u) P_{m}^{\prime}(u)\right) S_{n}(u), \\
K_{m n} & =\int d u d(u) S_{m}(u) S_{n}(u),
\end{aligned}
$$

and used the orthonormality condition in the pseudoscalar sector

$$
\int d u a(u) S_{m}(u) S_{n}(u)=\frac{1}{2} \lambda_{m}^{\phi} \delta_{m n}
$$

In addition, we choose the basis functions $\left\{P_{m}(u)\right\}$ to satisfy the eigenvalue equations

$$
-R^{6}\left(d(u) P_{m}^{\prime}(u)\right)^{\prime}+\left(b(u)+\frac{1}{2} R^{3} e^{\prime}(u)\right) P_{m}(u)=2 \lambda_{m}^{A} c(u) P_{m}(u) .
$$

Using these we see that

$$
L_{m n}^{A}=\frac{1}{2}\left[R^{6}\left(d(u) P_{m}^{\prime}(u) P_{n}(u)\right)_{\partial u}+2 \lambda_{m}^{A} Q_{m n}^{A}\right]+m \leftrightarrow n,
$$

where, as before, $\partial u$ refers to boundaries in the $u$-direction. 
We note that because of the last term in (5.17), the longitudinal component of $A_{\mu}^{(m)}$ and $\phi^{(m)}$ mix. So we need to define new field variables in terms of which the action (5.17) is diagonal. Before we do that, let us first note that the axial vector potential $A_{\mu}(x, u)$ has a possible zero mode provided the corresponding $(3+1)$ dimensional field strength vanishes, for reasons explained in the previous subsection. Hence the zero mode, which we shall denote by $A_{\mu}^{(0)}$, can only have a longitudinal component. The zero mode is gauge-invariant and, because of its mixing with the pseudoscalars, plays a special role. Let us see this in some detail.

The zero mode $A_{\mu}^{(0)}$ is conjugate to the eigenfunction $P_{0}(u)$ which satisfies the equation

$$
-R^{6}\left(d(u) P_{0}^{\prime}(u)\right)^{\prime}+\left(b(u)+\frac{1}{2} R^{3} e^{\prime}(u)\right) P_{0}(u)=0
$$

If there is no solution to this equation, then the zero mode does not exist and we should proceed directly to diagonalize the action (5.17). If, however, a solution $P_{0}(u)$ to this equation exists and is such that it goes to a constant at infinity, then the zero mode $A_{\mu}^{(0)}$ exists. Since it is purely longitudinal, for a reason identical to that discussed in the vector case, we make this explicit by writing it in terms of a pseudoscalar field, $A_{\mu}^{(0)}=\partial_{\mu} \phi^{(0)}(x)$. The terms in the action (5.17) which contain $\phi^{(0)}(x)$ can be separated out. These terms are:

$$
L_{00}^{A} \partial_{\mu} \phi^{(0)} \partial^{\mu} \phi^{(0)}+\sum_{m} J_{0 m} \partial_{\mu} \phi^{(m)} \partial^{\mu} \phi^{(0)}
$$

The sums over the indices $m, n$ no longer include the zero mode. Also, we have used $L_{m 0}^{A}=L_{0 m}^{A}=0$ for $m \neq 0$, which follows from (5.21) using the fact that $\lambda_{0}^{A}=0$ and the boundary terms vanish because $P_{m}(u)$ vanishes sufficiently fast at infinity. Without loss of generality, we may choose $L_{00}^{A}=1 / 2$ (to get the normalization of the kinetic term of $\phi^{(0)}$ right). Then, we can rewrite the above as

$$
\frac{1}{2} \partial_{\mu} \pi \partial^{\mu} \pi-\frac{1}{2} \sum_{m, n} J_{0 m} J_{0 n} \partial_{\mu} \phi^{(m)} \partial^{\mu} \phi^{(n)},
$$

where $\pi \equiv\left(\phi^{(0)}+\sum_{m} J_{0 m} \phi^{(m)}\right)$.

With the zero modes explicitly separated out in this way, for the nonzero modes we may, without loss of generality, choose

$$
Q_{m n}^{A}=\frac{1}{4} \delta_{m n}
$$

which, on using (5.21), gives

$$
L_{m n}^{A}=\frac{1}{2} \lambda_{m}^{A} \delta_{m n}
$$

Putting (5.23), (5.24) and (5.25) in the action (5.17), we get

$$
\begin{aligned}
\Delta S_{\text {gauge }}^{A}= & -\int d^{4} x\left[\sum_{m}\left(\frac{1}{2} \lambda_{m}^{\phi} \phi^{(m)} \phi^{(m)}+\frac{1}{4} F_{\mu \nu}^{A(m)} F^{A(m) \mu \nu}+\frac{1}{2} \lambda_{m}^{A} A_{\mu}^{(m)} A^{(m) \mu}\right)\right. \\
& \left.+\frac{1}{2} \partial_{\mu} \pi \partial^{\mu} \pi+\sum_{m, n}\left(\tilde{K}_{m n} \partial_{\mu} \phi^{(m)} \partial^{\mu} \phi^{(n)}+J_{m n} A^{(m) \mu} \partial_{\mu} \phi^{(n)}\right)\right]
\end{aligned}
$$


where $\tilde{K}_{m n}=\left(K_{m n}-\frac{1}{2} J_{0 m} J_{0 n}\right)$. The above action describes a massless particle, $\pi$, besides other massive particles. The existence of this massless particle depends on the existence of a solution to the equation (5.22), satisfying the normalization condition

$$
R^{6}\left(d(u) P_{0}(u) P_{0}^{\prime}(u)\right)_{\partial u}=\frac{1}{2} .
$$

Later we will see that the existence of the desired solution $P_{0}(u)$ depends on the absence of a non-normalizable part in $T(u)$.

To diagonalize the action (5.26) for the massive modes, we define the new variables

$$
A_{\mu}^{(m)}=\tilde{A}_{\mu}^{(m)}-\sum_{n}\left(\lambda_{m}^{A}\right)^{-1} J_{m n} \partial_{\mu} \phi^{(n)} .
$$

Putting in $(5.26)$, we get

$$
\begin{aligned}
\Delta S_{\text {gauge }}^{A}= & -\int d^{4} x\left[\sum_{m}\left(\frac{1}{2} \lambda_{m}^{\phi} \phi^{(m)} \phi^{(m)}+\frac{1}{4} F_{\mu \nu}^{A(m)} F^{A(m) \mu \nu}+\frac{1}{2} \lambda_{m}^{A} \tilde{A}_{\mu}^{(m)} \tilde{A}^{(m) \mu}\right)\right. \\
& \left.+\frac{1}{2} \partial_{\mu} \pi \partial^{\mu} \pi+\sum_{m, n} K_{m n}^{\prime} \partial_{\mu} \phi^{(m)} \partial^{\mu} \phi^{(n)}\right],
\end{aligned}
$$

where $K_{m n}^{\prime}=\left(\tilde{K}_{m n}-\frac{1}{2} \sum_{p}\left(\lambda_{p}^{A}\right)^{-1} J_{p m} J_{p n}\right)$. The modes have now been decoupled. To get the standard action for massive pseudoscalars we may, without loss of generality, set

$$
K_{m n}^{\prime}=\frac{1}{2} \delta_{m n}=K_{m n}-\frac{1}{2} J_{0 m} J_{0 n}-\frac{1}{2} \sum_{p}\left(\lambda_{p}^{A}\right)^{-1} J_{p m} J_{p n}
$$

This condition can be rewritten in a more conventional form as follows. We define

$$
\psi_{m}(u) \equiv \sum_{n}\left(\lambda_{n}^{A}\right)^{-1} P_{n}(u) J_{n m}+P_{0}(u) J_{0 m},
$$

and using (5.20) note that it satisfies the equation

$$
-R^{6}\left(d(u) \psi_{m}^{\prime}(u)\right)^{\prime}+\left(b(u)+\frac{1}{2} R^{3} e^{\prime}(u)\right) \psi_{m}(u)=\frac{1}{2} e(u) S_{m}(u)+R^{3}\left(d(u) S_{m}(u)\right)^{\prime} .
$$

Using (5.31) in (5.30), we get

$$
\delta_{m n}=\int d u\left(d(u) S_{m}(u)\left(S_{n}(u)+R^{3} \psi_{n}^{\prime}(u)\right)-\frac{1}{2} e(u) S_{m}(u) \psi_{n}(u)\right)+m \leftrightarrow n .
$$

In terms of new variables defined by

$$
S_{m}(u) \equiv R^{3} \eta_{m}^{\prime}(u), \quad \theta_{m}(u) \equiv \psi_{m}(u)+\eta_{m}(u)
$$

(5.33) can be written as

$$
\int d u \eta_{m}^{\prime}(u)\left(R^{6} d(u) \theta_{n}^{\prime}(u)-\frac{1}{2} R^{3} e(u)\left(\theta_{n}(u)-\eta_{n}(u)\right)\right)+m \leftrightarrow n=\delta_{m n} .
$$


Moreover, in terms of these variables the differential equation (5.32) reads

$$
-R^{6}\left(d(u) \theta_{m}^{\prime}(u)\right)^{\prime}+\left(b(u)+\frac{1}{2} R^{3} e^{\prime}(u)\right)\left(\theta_{m}(u)-\eta_{m}(u)\right)-\frac{1}{2} R^{3} e(u) \eta_{m}^{\prime}(u)=0,
$$

From these two equations one can obtain the orthonormality condition

$$
\begin{aligned}
& \int d u\left(R^{6} d(u) \theta_{m}^{\prime}(u) \theta_{n}^{\prime}(u)+\left(b(u)+\frac{1}{2} R^{3} e^{\prime}(u)\right)\left(\theta_{m}(u)-\eta_{m}(u)\right)\left(\theta_{n}(u)-\eta_{n}(u)\right)\right. \\
& \left.-\frac{1}{2} R^{3} e(u) \eta_{m}^{\prime}(u)\left(\theta_{n}(u)-\eta_{n}(u)\right)-\frac{1}{2} R^{3} e(u) \eta_{n}^{\prime}(u)\left(\theta_{m}(u)-\eta_{m}(u)\right)\right)=\frac{1}{2} \delta_{m n} .
\end{aligned}
$$

Also, rewriting (5.19) in terms of the new variables, we have

$$
R^{6} \int d u a(u) \eta_{m}^{\prime}(u) \eta_{n}^{\prime}(u)=\frac{1}{2} \lambda_{m}^{\phi} \delta_{m n}
$$

Finally, (5.35) and (5.38) give

$$
R^{6} a(u) \eta_{n}^{\prime}(u)=\lambda_{n}^{\phi}\left(R^{6} d(u) \theta_{n}^{\prime}(u)-\frac{1}{2} R^{3} e(u)\left(\theta_{n}(u)-\eta_{n}(u)\right)\right) .
$$

Equations (5.36) and (5.39) are the final form of the eigenvalue equations and (5.37) and $(5.38)$ are the orthonormality conditions in the pseudoscalar sector.

It is interesting to note from (5.36) that if $\eta$ is constant, then the variable $(\theta-\eta)$ satisfies a differential equation that is identical to the equation (5.22) satisfied by the zero mode $P_{0}$. Also, using (5.36) and (5.37) one can show that for constant $\eta$, $(\theta-\eta)$ satisfies the normalization condition (5.27). From (5.39) it follows that if $\eta$ is constant, the eigenvalue $\lambda^{\phi}$ vanishes. Thus, the presence of a massless pseudoscalar can be naturally considered to be identical to the question of the existence of a solution to the equations (5.36)-(5.39) with zero eigenvalue, and so it becomes a part of the spectrum in the pseudoscalar tower of states. Hence, the action in this sector can be written in the form

$$
\begin{aligned}
\Delta S_{\text {gauge }}^{A}=-\int d^{4} x \sum_{m}\left[\frac{1}{4} F_{\mu \nu}^{A(m)} F^{A(m) \mu \nu}+\frac{1}{2} \lambda_{m}^{A} \tilde{A}_{\mu}^{(m)} \tilde{A}^{(m) \mu}\right. \\
\left.+\frac{1}{2} \partial_{\mu} \phi^{(m)} \partial^{\mu} \phi^{(m)}+\frac{1}{2} \lambda_{m}^{\phi} \phi^{(m)} \phi^{(m)}\right] .
\end{aligned}
$$

Note that we have dropped the field $\pi(x)$, but extended the sum over $m$ to cover a possible zero mode as well. If there is a solution to the equations (5.36)-(5.39) with constant $\eta_{0}$ and $\lambda_{0}^{\phi}=0$, then a massless pion field will reappear as the zero mode $\phi^{(0)}$ in the pseudoscalar tower. Otherwise, the lowest mode in this sector will be massive, whose mass can be computed as in the following subsection. 


\subsection{Relation between pion mass and non-normalizable part of tachyon}

In this subsection we will derive a relation between the pion mass and the nonnormalizable part of tachyon parametrized by $T_{-}$. This will give us further evidence for identifying the parameters $T_{+}$and $T_{-}$with the chiral condensate and quark mass respectively. We first note that for $T(u)=0, a(u)$ vanishes and hence $\lambda_{m}^{\phi}$ also vanishes by (5.39). However, as we will see from the following calculations, $T(u)=0$ is a sufficient condition, but not necessary to guarantee the presence of a massless pion. The necessary condition is that the non-normalizable piece in $T(u)$ should be absent, i.e. $T_{-}=0$.

Let us assume that $T(u) \neq 0$ so that $a(u) \neq 0$. Then, (5.39) can be used to solve for $\eta_{m}(u)$ in terms of $\psi_{m}(u)$, which is related to $\theta_{m}(u)$ and $\eta_{m}(u)$ by (5.34). We get,

$$
\eta_{m}^{\prime}(u)=\frac{\lambda_{m}^{\phi}}{a(u)-\lambda_{m}^{\phi} d(u)}\left(d(u) \psi_{m}^{\prime}(u)-\frac{e(u)}{2 R^{3}} \psi_{m}(u)\right)
$$

Let us now denote by $\lambda_{0}^{\phi}$ the lowest mass eigenvalue. The corresponding eigenfunctions are $\psi_{0}(u)$ and $\eta_{0}(u)$. Assuming $\lambda_{0}^{\phi} \ll a(u) / d(u)^{23}$, we can approximate the above equation for $\eta_{0}(u)$ :

$$
\eta_{0}^{\prime}(u) \approx \frac{\lambda_{0}^{\phi}}{a(u)}\left(d(u) \psi_{0}^{\prime}(u)-\frac{e(u)}{2 R^{3}} \psi_{0}(u)\right)
$$

If we know $\psi_{0}(u)$, then using the above in (5.38) we can compute the mass. Now, $\psi_{0}(u)$ satisfies the following differential equation, which can be obtained from (5.36) using (5.42) and the approximation $\lambda_{0}^{\phi} \ll a(u) / d(u)$ :

$$
-R^{6}\left(d(u) \psi_{0}^{\prime}(u)\right)^{\prime}+\left(b(u)+\frac{1}{2} R^{3} e^{\prime}(u)\right) \psi_{0}(u) \approx 0 .
$$

Also, using (5.43) and the approximation under which it was obtained, the normalization condition on $\psi_{0}(u)$ given by (5.37) can be approximated as

$$
\left.R^{6} d(u) \psi_{0}^{\prime}(u) \psi_{0}(u)\right|_{u=u_{\max }} \approx \frac{1}{2} .
$$

These equations cannot be solved analytically in general. However, analytic solutions can be obtained in the IR and UV regimes. In the UV regime, for $u \lesssim u_{\max }$, we use (2.16) and (2.18) to approximate the coefficients in (5.43); we get

$$
b(u) \approx \frac{V_{4} V(0)}{R^{3}} u T^{2}(u), \quad d(u) \approx \frac{V_{4} V(0)}{4 R^{9}} u^{5 / 2}, \quad e(u) \approx \frac{9 V_{4} V(0)}{4 R^{6}} h_{0} h_{1} u^{-3 / 2} T^{2}(u) .
$$

\footnotetext{
${ }^{23}$ This approximation can be justified a posteriori by the solution because the eigenvalue $\lambda_{0}^{\phi}$ turns out to be parametrically much smaller by a factor of $1 / R^{3}$, see (5.56), compared to the ratio $a(u) / d(u)$.
} 
In writing these, we have used $f(u) \approx 1$, which is a good approximation for large $u$. We see that we can clearly neglect $e(u)$ compared to $b(u)$ in (5.43), while $b(u)$ is itself negligible compared to $d(u)$. Using these approximations in (5.43) and (5.44) then gives

$$
-\left(u^{\frac{5}{2}} \psi_{0}^{\prime}(u)\right)^{\prime} \approx 0,\left.\quad \frac{V_{4} V(0)}{4 R^{3}} u^{\frac{5}{2}} \psi_{0}^{\prime}(u) \psi_{0}(u)\right|_{u=u_{\max }} \approx \frac{1}{2},
$$

which are solved by

$$
\psi_{0}(u) \approx c_{0}-\frac{1}{3 c_{0}} \frac{4 R^{3}}{V_{4} V(0)} u^{-3 / 2} .
$$

Here $c_{0}$ is a parameter which is related to the pion decay constant. This can be argued by analysing the 4-d axial current correlator and using AdS/CFT along the lines of [45, 46]. Using the AdS/CFT dictionary, one can compute the axial current correlator from the action (5.17), evaluated on-shell, by differentiating twice with respect to the transverse part of the axial vector field on the UV boundary. This is the source which couples to the axial current on the boundary. The source arises from the same zero mode solution, $P_{0}(u)$, which we discussed in connection with a possible zero mode (the pion) in the longitudinal component of the axial gauge field. $P_{0}(u)$ satisfies the equation (5.22), which is identical to that satisfied by $\psi_{0}(u)$, (5.43). However, the boundary condition now is different; it is the boundary condition for a source, $P_{0}\left(u_{\max }\right)=1$. In addition, one imposes the condition

$$
\left.R^{6} d(u) P_{0}^{\prime}(u) P_{0}(u)\right|_{u=u_{\max }} \approx \frac{f_{\pi}^{2}}{2},
$$

which is required to reproduce the correct zero momentum axial current correlator [45, 46]. This follows from the action (5.17). Now, $P_{0}(u)$ satisfies (5.22) and the condition (5.48) if we set $P_{0}(u)=f_{\pi} \psi_{0}(u)$. Then, requiring $P_{0}\left(u_{\max }\right)=1$ gives $c_{0}=1 / f_{\pi}$.

In the IR regime, $u \gtrsim u_{0}$, we use (2.21) and (2.22) to approximate the coefficients in (5.43); we get

$$
b(u) \approx \frac{\pi^{3 / 2} V_{4} u_{0}^{17 / 4}}{26 R^{3}} \frac{V(T)}{\left(u-u_{0}\right)^{4}}, \quad d(u) \approx \frac{13 V_{4} u_{0}^{9 / 4}}{32 \sqrt{\pi} R^{9}} V(T), \quad e(u) \approx \frac{13 V_{4} u_{0}^{9 / 4}}{16 \sqrt{\pi} R^{6}} \frac{V(T)}{\left(u-u_{0}\right)} .
$$

In writing these, we have used $f\left(u_{0}\right) \approx 1$, which is a good approximation for large $u_{0}$. Using $d V(T) / d u=T^{\prime}(u) V^{\prime}(T)$, we see that $b(u)$ and $R^{3} e^{\prime}(u)$ both go as $\left(u-u_{0}\right)^{-4}$ in this regime. However, the coefficient of the latter is suppressed by a relative factor of $u_{0}^{-1 / 2}$, so for large $u_{0}$ we may neglect it compared to $b(u)$. But, unlike in the UV regime, $b(u)$ cannot be neglected compared to $d(u)$. In fact, this term is crucial for getting a nontrivial solution. In this regime, then, the leading terms in equation (5.43) give

$$
\psi_{0}^{\prime}(u) \approx \frac{32 \pi R^{6} u_{0}^{1 / 2}}{169} \frac{\psi_{0}(u)}{\left(u-u_{0}\right)}
$$


which has the solution

$$
\psi_{0}(u) \approx \tilde{c_{0}}\left(u-u_{0}\right)^{\frac{32 \pi R^{6} u_{0}^{1 / 2}}{169}},
$$

where $\tilde{c_{0}}$ is an integration constant. Note that the normalization condition remains unchanged and cannot be used here because it receives contribution only from the UV end due to the exponentially vanishing tachyon potential for large $T(u)$ at the IR end.

Let us now consider the formula, (5.38), for the lowest mode, using which one can compute the eigenvalue $\lambda_{0}^{\phi}$ :

$$
R^{6} \int_{u_{0}}^{u_{\max }} d u a(u)\left(\eta_{0}^{\prime}(u)\right)^{2}=\frac{1}{2} \lambda_{0}^{\phi} .
$$

Using $a(u) \approx \frac{\sqrt{\pi} V_{4} u_{0}^{19 / 4}}{8 R^{15}} \frac{V(T)}{\left(u-u_{0}\right)}$ in the IR and (5.51) in (5.42), we see that $\eta_{0}^{\prime}(u) \propto \psi_{0}(u)$ vanishes very rapidly as $u \rightarrow u_{0}$, with a power which grows as $u_{0}^{1 / 2}$ for large $u_{0}$. Moreover, since $V(T)$ vanishes exponentially for large $T$, the IR region makes a negligible contribution to the integral. Therefore, it is reasonable to calculate the integral by substituting the UV estimate of the integrand in it. In the UV region, $a(u) \approx \frac{V_{4} V(0)}{R^{15}} u^{4} T^{2}(u)$. Moreover, in this region the second term on the right hand side of (5.42) can be neglected. So, we get

$$
\begin{aligned}
\frac{1}{2} \lambda_{0}^{\phi}=R^{6} \int_{u_{0}}^{u_{\max }} d u a(u)\left(\eta_{0}^{\prime}(u)\right)^{2} & \approx R^{6}\left(\lambda_{0}^{\phi}\right)^{2} \int_{\tilde{u}_{0}}^{u_{\max }} d u \frac{d^{2}(u)}{a(u)}\left(\psi_{0}^{\prime}(u)\right)^{2} \\
& \approx\left(\lambda_{0}^{\phi}\right)^{2} \kappa \int_{\tilde{u}_{0}}^{u_{\max }} \frac{h_{0} d u}{\left(T_{+} e^{-h_{0} u}+T_{-} e^{h_{0} u}\right)^{2}},
\end{aligned}
$$

where $\tilde{u}_{0}>u_{0}$ avoids the IR region in the integral and we have defined

$$
\kappa \equiv \frac{f_{\pi}^{2} R^{9}}{4 h_{0} V_{4} V(0)} .
$$

The integral is easily done, giving

$$
\lambda_{0}^{\phi} \approx \frac{1}{\kappa} \frac{\left(T_{+} e^{-h_{0} \tilde{u}_{0}}+T_{-} e^{h_{0} \tilde{u}_{0}}\right)\left(T_{+} e^{-h_{0} u_{\max }}+T_{-} e^{h_{0} u_{\max }}\right)}{e^{h_{0}\left(u_{\max }-\tilde{u}_{0}\right)}-e^{-h_{0}\left(u_{\max }-\tilde{u}_{0}\right)}} .
$$

From our numerical solutions we see that it is possible to choose $\tilde{u}_{0}$ to be relatively large and also satisfy the conditions $\left|T_{+}\right| e^{-h_{0} \tilde{u}_{0}} \gg\left|T_{-}\right| e^{h_{0} \tilde{u}_{0}}$ and $e^{h_{0}\left(u_{\max }-\tilde{u}_{0}\right)} \gg$ $e^{-h_{0}\left(u_{\max }-\tilde{u}_{0}\right)}$. For such values of the parameters, then, to a good approximation (5.54) gives

$$
\lambda_{0}^{\phi} \approx \frac{1}{\kappa}\left(T_{+} T_{-}+T_{+}^{2} e^{-2 h_{0} u_{\max }}\right) .
$$

Now, let us tune $u_{\max }$ to large values. We will do this in a manner consistent with the inequality (2.19). As explained in section 2.4, one way of maintaining this inequality is to keep $\left|T_{+}\right|$and $\left|T_{-}\right| e^{h_{0} u_{\max }}$ fixed as $u_{\max }$ becomes large. In that case, 
the second term on the right hand side of (5.55) becomes exponentially smaller than the first term as the cut-off is increased beyond some value. We may then neglect this term compared with the first term. This gives

$$
\lambda_{0}^{\phi} \approx \frac{1}{\kappa} T_{+} T_{-} .
$$

Finally, using $\lambda_{0}^{\phi}=m_{\pi}^{2}$ and (4.23) in this relation, we get

$$
m_{\pi}^{2} \approx \frac{m_{q} \chi}{f_{\pi}^{2}},
$$

This is the well-known Gell-Mann-Oakes-Renner formula, up to a factor of 2 .

\section{Summary and Discussion}

This paper further explores our proposal [21] of a modified SS model, which includes the degree of freedom associated with the open string tachyon between the flavour branes and antibranes. Here we have extended the analytic treatment of various aspects of the problem and supplemented it with extensive numerical calculations. We have argued that taking the tachyon into account is essential for the consistency of the setup and shown numerically that the solution which includes the tachyon is energetically favoured. Our modification preserves the nice geometric picture of chiral symmetry breaking of the SS model and at the same time relates chiral symmetry breaking to tachyon condensation; the tachyon becomes infinitely large in the infrared region where the joining of the flavour branes signals chiral symmetry breaking.

We have identified a parameter in the non-normalizable part of the tachyon field profile with the quark mass. It is important to stress that this is the only tunable parameter in the modified SS model. It can be traded for the asymptotic brane-antibrane separation or the location of the point in the bulk where the brane and antibrane join. This provides a natural explanation for the latter parameter, which is also present in the SS model, but in that model it doesn't find any counterpart in the QCD-like theory at the boundary. In this paper we have presented numerical evidence to show that the point where the brane and antibrane meet is monotonically shifted towards ultraviolet as we tune the mass parameter to larger values. It would seem, therefore, that in our model a brane-antibrane pair disappears from the bulk consistently with a quark flavour becoming infinitely massive.

The presence of a non-normalizable part in the tachyon solution requires us to introduce an ultraviolet cut-off. The cut-off is needed not only because this part grows as one moves towards the ultraviolet region, as in any standard AdS/CFT example that includes a non-normalizable solution, but also because the asymptotic form of the solution is derived from an approximate equation which is valid only for 
small values of the tachyon. Therefore, the asymptotic solution itself is not valid beyond a certain maximum value of the holographic coordinate. We have presented sufficient numerical evidence of this phenomenon. Removing the ultraviolet cut-off, then, requires tuning the mass parameter to zero. We have explained one scheme by which this can be done. This scheme gives an exponential dependence on the cut-off to the mass parameter, similar to that discussed recently in [29]. The quark mass arises from an apparently very different mechanism in this work and the cut-off is related to the location of a $D 6$-brane that is present in this model. It would be interesting to see if there is any connection between this model and our model.

Once we have identified the quark mass as a parameter in the non-normalizable part of the tachyon, it is natural to expect, by the usual AdS/CFT rules, the normalizable part of the tachyon solution to give rise to the chiral condensate. To derive an expression for it, however, we need to go beyond the space-time independent solutions of section 2. As we have seen, this requires an exact expression for the 5-dimensional action for tachyon and brane-antibrane separation fields which are now taken to depend on space-time as well as the holographic coordinate. We have derived this action in this paper. Using the generalized solutions to the equations for this action, then, one can compute the chiral condensate. However, one also needs to add counter terms to the boundary brane action to remove from it contributions that diverge when the cut-off is removed.

We have studied in detail the fluctuations of flavour gauge fields on the braneantibrane system. These give rise to vector, axial vector and pseudoscalar towers of mesons, which become massive through a kind of higgs mechanism, except for the pions. These arise from a gauge-invariant combination of the tachyon phase and the longitudinal zero mode of the axial vector field. We have shown that the pions remain massless, unless a quark mass (non-normalizable part of the tachyon solution) is switched on. For small quark mass, we have derived an expression for the mass of the lowest pseudoscalar meson in terms of the chiral condensate and shown that it satisfies the Gell-Mann-Oakes-Renner relation. The vector and axial vector spectra are expected to be non-degenerate because they arise from eigenvalue equations with different tachyon contributions. We have not computed these spectra, but it would be interesting to see whether they have the Regge behaviour for large masses.

A non-zero quark mass is essential to correctly reproduce phenomenology in the low-energy sector of QCD. Therefore, our modified SS model can be the starting point of a more quantitative version of the phenomenology initiated in [1]. For this purpose, our treatment needs to be extended to the non-abelian case, which should be a straightforward exercise. The correct tachyon brane-antibrane action for curved directions transverse to the branes is not known. It is important to have such an action since this would extend the applicability of the present treatment to such interesting cases as e.g. the antipodal configuration of the flavour brane system 
and its connection with massless quarks. Another direction in which the present ideas can be extended is to discuss this model at finite temperature and describe the chiral symmetry restoration transition and study the phase diagram in some detail. The connection of chiral symmetry breaking with tachyon condensation seems fascinating and a deeper understanding would be useful. Finally, baryons have been discussed in the SS model. It turns out that they have a very small size. This may change in the presence of the tachyon. This is because in the presence of the tachyon, the flavour energy momentum tensor is concentrated away from the infrared region where the branes meet. In other words, there is a new scale provided by the quark mass. It would be very interesting to investigate whether this effect makes any difference to the baryon size.

\section{Acknowledgment}

It is a pleasure to thank Gautam Mandal and Spenta Wadia for discussions.

\section{A. Overlapping $D 8-\bar{D} 8$-brane system}

In this case the appropriate DBI action is

$$
\begin{gathered}
S=-\int d^{9} \sigma g_{s} V(T) e^{-\phi}\left(\sqrt{-\operatorname{det} \mathrm{A}_{\mathrm{L}}}+\sqrt{-\operatorname{det} \mathrm{A}_{\mathrm{R}}}\right), \\
\left(A_{i}\right)_{a b}=g_{M N} \partial_{a} x_{i}^{M} \partial_{b} x_{i}^{N}+F_{a b}^{i}+\frac{1}{2}\left(\left(D_{a} \tau\left(D_{b} \tau\right)^{*}+\left(D_{a} \tau\right)^{*} D_{b} \tau\right)\right),
\end{gathered}
$$

where $D_{a} \tau=\partial_{a} \tau-i\left(A_{L, a}-A_{R, a}\right) \tau$. The classical equation for the profile of the magnitude $T$ of the tachyon $\tau$ can be obtained from (2.13) by substituting $h=0$ in it everywhere. We get

$$
\left(\frac{u^{\frac{13}{4}}}{\sqrt{d_{T}}} T^{\prime}(u)\right)^{\prime}=\frac{u^{\frac{7}{4}} f(u)^{-1}}{\sqrt{d_{T}}} \frac{V^{\prime}(T)}{V(T)},
$$

where now $d_{T}=f(u)^{-1} u^{-3 / 2}+T^{\prime}(u)^{2}$. In the UV region, assuming $T$ is small for large $u$, we can approximate this equation as

$$
\left(u^{4} T^{\prime}(u)\right)^{\prime}=-\pi u^{5 / 2} T(u)
$$

where we have used the universal small $T$ expansion, $V(T)=\mathcal{T}_{8}\left(1-\frac{\pi}{2} T^{2}+\cdots\right)$. The general solution ${ }^{24}$ to this equation is

$$
T(u)=u^{-13 / 8}\left(c_{1} \cos \left(4 \sqrt{\pi} u^{1 / 4}\right)+c_{2} \sin \left(4 \sqrt{\pi} u^{1 / 4}\right)\right)+\cdots,
$$

\footnotetext{
${ }^{24}$ Equation $($ A.3 $)$ can be solved exactly in terms of the Bessel functions $H^{(1)}$ and $H^{(2)}$. Here we give only the leading term.
} 
where $c_{1}$ and $c_{2}$ are arbitrary constants. Both the independent solutions in this case are normalizable, so the interpretation of one of the parameters corresponding to a source for the quark mass term is not clear. In view of this, it is not clear how to apply the general treatment of [19 to this case.

In the IR region, a singular tachyon solution is obtained only for $u \sim u_{k}$. In this region $f(u)^{-1}$ blows up as $\left(u-u_{k}\right)^{-1}$ and this drives a singularity in the tachyon. Both the potentials in (2.5) and (2.6) exhibit singular solutions, although the solutions and the nature of singularity are different. For the potential (2.5) we find the solution

$$
T(u)=\left(\pi+\frac{39}{2 \sqrt{u_{k}}}\right)^{-1 / 2} \ln \frac{1}{\left(u-u_{k}\right)}+b_{1}+\cdots,
$$

while for (2.6) we get

$$
T(u)=b_{2}\left(u-u_{k}\right)^{-\alpha}+\cdots,
$$

where $b_{1}$ and $b_{2}$ are arbitrary constants and $\alpha=\frac{4 \pi \sqrt{u_{k}}}{39}$. As in the case with nonzero brane-antibrane separation, the IR solution for which the tachyon blows up exhibits a smaller number of independent parameters than the UV solution, one in the IR as opposed to two in the UV in the present case. A solution with two independent parameters in the IR exists (for any potential), but this solution is finite:

$$
T(u)=T_{0}+T_{1}\left(u-u_{k}\right)^{1 / 2}+\left(\frac{2}{3 \sqrt{u_{k}}}+\frac{T_{1}^{2}}{2}\right) \frac{V^{\prime}\left(T_{0}\right)}{V\left(T_{0}\right)}\left(u-u_{k}\right)+\cdots .
$$

Here $T_{0}$ and $T_{1}$ are the two arbitrary parameters.

\section{B. Scalar fluctuations}

Here we will assume that $T(u, x)$ and $h(u, x)$ are weakly dependent on $x^{\mu}$ and expand $\operatorname{det}(1+K)$ in (4.1) in powers of space-time derivatives. The action correct to quadratic terms in the derivatives is

$$
\begin{aligned}
S= & -\frac{2 V_{4}}{R^{9}} \int d^{4} x \int d u u^{13 / 4} V(T) \sqrt{d_{T}}\left[1+\frac{u^{-3 / 2}}{2 Q}\left\{\left(1-\frac{T^{\prime 2}}{d_{T}}\right)(\partial T)^{2}\right.\right. \\
& \left.\left.+\left(1-\frac{\frac{1}{4} f u^{3 / 2} h^{\prime 2}}{d_{T}}\right) \frac{1}{4} f u^{3 / 2}(\partial h)^{2}-\frac{f u^{3 / 2} h^{\prime} T^{\prime}}{2 d_{T}}(\partial h) .(\partial T)\right\}\right]
\end{aligned}
$$

where $d_{T}$ is given by (2.11), with $T(u)$ replaced by $T(u, x)$ and $h(u)$ by $h(u, x)$. Also, the notation $(\partial T)^{2}$ stands for $\eta^{\mu \nu} \partial_{\mu} T(u, x) \partial_{\nu} T(u, x)$; similar expressions hold for $(\partial h)^{2}$ and $(\partial h) .(\partial T)$. For the expansion in derivatives to be valid, we must require the following conditions to be satisfied: (i) For large values of $u$, near the cut-off $u_{\max }$, we must have $|\partial T|<<u_{\max }^{3 / 4}$ and $|\partial h|<<1$; (ii) For $u \sim u_{0}$, we must have $|\partial T|<<\left|T^{\prime}\right| \sim\left(u-u_{0}\right)^{-3}$ and $|\partial h|<<|h T| \sim\left(u-u_{0}\right)^{-3 / 2}$. 
Let us now consider small fluctuations around the $x$-independent solutions. We write $T(u, x)=T_{c}(u)+T_{q}(u, x)$ and $h(u, x)=h_{c}(u)+h_{q}(u, x)$, where $T_{c}(u)$ and $h_{c}(u)$ are the $x$-independent solutions of the classical equations (2.13), (2.14). We now expand the above action and retain only terms up to second order in the fluctuations $T_{q}(u, x)$ and $h_{q}(u, x)$. We get

$$
\begin{aligned}
S=-\frac{2 V_{4}}{R^{9}} \int & d^{4} x \int_{u_{0}}^{\infty} d u A \sqrt{d_{c}}\left[1+\left\{\frac{V_{c}^{\prime}}{V_{c}} T_{q}+\frac{1}{d_{c}}\left(\frac{1}{4} f u^{3 / 2} h_{c}^{\prime} h_{q}^{\prime}+T_{c}^{\prime} T_{q}^{\prime}+h_{c} T_{c}^{2} h_{q}+h_{c}^{2} T_{c} T_{q}\right)\right\}\right. \\
+ & \left\{\frac{V_{c}^{\prime \prime}}{2 V_{c}} T_{q}^{2}+\frac{V_{c}^{\prime}}{V_{c} d_{c}}\left(\frac{1}{4} f u^{3 / 2} h_{c}^{\prime} h_{q}^{\prime} T_{q}+T_{c}^{\prime} T_{q}^{\prime} T_{q}+h_{c} T_{c}^{2} h_{q} T_{q}+h_{c}^{2} T_{c} T_{q}^{2}\right)\right. \\
& +\frac{1}{2 d_{c}}\left(\frac{1}{4} f u^{3 / 2} h_{q}^{\prime 2}+T_{q}^{\prime 2}+T_{c}^{2} h_{q}^{2}+h_{c}^{2} T_{q}^{2}+4 h_{c} T_{c} h_{q} T_{q}\right)-\frac{1}{2 d_{c}^{2}}\left(\frac{1}{4} f u^{3 / 2} h_{c}^{\prime} h_{q}^{\prime}\right. \\
& \left.+T_{c}^{\prime} T_{q}^{\prime}+h_{c} T_{c}^{2} h_{q}+h_{c}^{2} T_{c} T_{q}\right)^{2}+\frac{u^{-3 / 2}}{2 Q_{0}}\left(\left(1-\frac{T_{c}^{\prime 2}}{d_{c}}\right)\left(\partial T_{q}\right)^{2}\right. \\
& \left.\left.\left.-\frac{f u^{3 / 2}}{2 d_{c}} h_{c}^{\prime} T_{c}^{\prime}\left(\partial h_{q}\right) \cdot\left(\partial T_{q}\right)+\left(1-\frac{\frac{1}{4} f u^{3 / 2} h_{c}^{\prime 2}}{d_{c}}\right) \frac{1}{4} f u^{3 / 2}\left(\partial h_{q}\right)^{2}\right)\right\}+\cdots\right],
\end{aligned}
$$

where we have used the notation $V_{c}=V\left(T_{c}\right), d_{c}=d_{T_{c}}$, and $A=u^{13 / 4} V_{c}$. As before, a prime denotes derivative w.r.t. $u$, except on $V_{c}$, for which it denotes a derivative w.r.t. its argument. The part of this action linear in fluctuations, $S_{1}$, which arises from the term in the first curly brackets above, is given by

$$
S_{1}=-\frac{2 V_{4}}{R^{9}} \int d^{4} x \int_{u_{0}}^{\infty} d u A\left[\frac{V_{c}^{\prime}}{V_{c}} \sqrt{d_{c}} T_{q}+\frac{1}{\sqrt{d_{c}}}\left(\frac{1}{4} f u^{3 / 2} h_{c}^{\prime} h_{q}^{\prime}+T_{c}^{\prime} T_{q}^{\prime}+h_{c} T_{c}^{2} h_{q}+h_{c}^{2} T_{c} T_{q}\right)\right],
$$

It is easy to verify that $S_{1}$ leads to the background equations (2.13) and (2.14). This part of the action, therefore, vanishes, except for a boundary term. It is this boundary term that gives rise to the chiral condensate.

The term in the second curly brackets becomes $S_{2}$, the action quadratic in fluctuations, after some manipulations. First, we open the square in the coefficient of $1 / 2 d_{c}^{2}$ term and combine it with the term just before it. That is, we have,

$$
\begin{aligned}
& \frac{1}{2 d_{c}}\left(\frac{1}{4} f u^{3 / 2} h_{q}^{\prime 2}+T_{q}^{\prime 2}+T_{c}^{2} h_{q}^{2}+h_{c}^{2} T_{q}^{2}+4 h_{c} T_{c} h_{q} T_{q}\right) \\
& -\frac{1}{2 d_{c}^{2}}\left(\frac{1}{4} f u^{3 / 2} h_{c}^{\prime} h_{q}^{\prime}+T_{c}^{\prime} T_{q}^{\prime}+h_{c} T_{c}^{2} h_{q}+h_{c}^{2} T_{c} T_{q}\right)^{2} \\
& =\frac{1}{2 d_{c}}\left\{\left(1-\frac{\frac{1}{4} f u^{3 / 2} h_{c}^{\prime 2}}{d_{c}}\right) \frac{1}{4} f u^{3 / 2} h_{q}^{\prime 2}+\left(1-\frac{T_{c}^{\prime 2}}{d_{c}}\right) T_{q}^{\prime 2}+\left(1-\frac{h_{c}^{2} T_{c}^{2}}{d_{c}}\right)\left(h_{c}^{2} T_{q}^{2}+T_{c}^{2} h_{q}^{2}\right)\right. \\
& \left.+2\left(2-\frac{h_{c}^{2} T_{c}^{2}}{d_{c}}\right) h_{c} T_{c} h_{q} T_{q}\right\}-\frac{1}{d_{c}^{2}}\left\{\frac{1}{4} f u^{3 / 2} h_{c}^{\prime}\left(T_{c}^{\prime} h_{q}^{\prime} T_{q}^{\prime}+T_{c}^{2} h_{c} h_{q} h_{q}^{\prime}+h_{c}^{2} T_{c} h_{q}^{\prime} T_{q}\right)\right. \\
& \left.+T_{c}^{\prime} h_{c} T_{c}\left(T_{c} T_{q}^{\prime} h_{q}+h_{c} T_{q}^{\prime} T_{q}\right)\right\}
\end{aligned}
$$

Furthermore, we can rewrite

$$
A \frac{V_{c}^{\prime}}{V_{c} \sqrt{d_{c}}} T_{c}^{\prime} T_{q} T_{q}^{\prime} \sim V_{c}^{\prime}\left(\frac{u^{13 / 4} T_{c}^{\prime}}{\sqrt{d_{c}}}\right)\left(\frac{T_{q}^{2}}{2}\right)^{\prime}
$$




$$
\rightarrow-A \sqrt{d_{c}}\left[\frac{V_{c}^{\prime \prime}}{V_{c}} \frac{T_{c}^{\prime 2}}{d_{c}}+\frac{V_{c}^{\prime}}{V_{c}}\left(\frac{h_{c}^{2} T_{c}}{d_{c}}+\frac{V_{c}^{\prime}}{V_{c}}\left(1-\frac{T_{c}^{2}}{d_{c}}\right)\right)\right] \frac{T_{q}^{2}}{2},
$$

where in the last step we have done an integration by parts over $u$, used the equation of motion (2.13) for $T_{c}, h_{c}$ and ignored a possible boundary term since it is quadratic in fluctuations and so will not contribute to the calculation of the condensate. A similar manipulation gives

$$
\begin{aligned}
-\frac{A}{d_{c} \sqrt{d_{c}}} T_{c}^{\prime} h_{c}^{2} T_{c} T_{q} T_{q}^{\prime} & \sim-V_{c}\left(\frac{u^{13 / 4} T_{c}^{\prime}}{\sqrt{d_{c}}}\right)\left(\frac{h_{c}^{2} T_{c}}{d_{c}}\right)\left(\frac{T_{q}^{2}}{2}\right)^{\prime} \\
& \rightarrow A \sqrt{d_{c}}\left[\left(\frac{V_{c}^{\prime}}{V_{c}}+\frac{h_{c}^{2} T_{c}}{d_{c}}\right) \frac{h_{c}^{2} T_{c}}{d_{c}}+\frac{T_{c}^{\prime}}{d_{c}}\left(\frac{h_{c}^{2} T_{c}}{d_{c}}\right)^{\prime}\right] \frac{T_{q}^{2}}{2} .
\end{aligned}
$$

Combining the above with the other three $T_{q}^{2} / 2$ terms, we find its net coefficient to be

$$
A\left\{\left(\frac{V_{c}^{\prime \prime}}{V_{c}}-\left(\frac{V_{c}^{\prime}}{V_{c}}\right)^{2}\right)\left(1-\frac{T_{c}^{\prime 2}}{d_{c}}\right) \sqrt{d_{c}}+2 \frac{V_{c}^{\prime}}{V_{c}} \frac{h_{c}^{2} T_{c}}{\sqrt{d_{c}}}+\frac{h_{c}^{2}}{\sqrt{d_{c}}}+\frac{T_{c}^{\prime}}{\sqrt{d_{c}}}\left(\frac{h_{c}^{2} T_{c}}{d_{c}}\right)^{\prime}\right\}
$$

Similarly, a partial integration using the equation of motion (2.14) allows us to combine the two $h_{q}^{2} / 2$ terms, giving its net coefficient to be

$$
A\left\{\left(\frac{h_{c} T_{c}^{2}}{d_{c}}\right)^{\prime} \frac{\frac{1}{4} f u^{3 / 2} h_{c}^{\prime}}{\sqrt{d_{c}}}+\frac{T_{c}^{2}}{\sqrt{d_{c}}}\right\}
$$

Collecting all this together, we get the action quadratic in fluctuations:

$$
\begin{aligned}
S_{2}= & -\frac{2 V_{4}}{R^{9}} \int d^{4} x \int_{u_{0}}^{\infty} d u A\left[\frac{1}{2} c_{1} T_{q}^{2}+\frac{1}{2} c_{2} h_{q}^{2}+\frac{1}{2} c_{3} h_{q}^{\prime 2}+\frac{1}{2} c_{4} T_{q}^{\prime 2}+c_{5} h_{q} T_{q}+c_{6} h_{q}^{\prime} T_{q}^{\prime}\right. \\
& \left.+c_{7} h_{q}^{\prime} T_{q}+c_{8} h_{q} T_{q}^{\prime}+\frac{c_{9}}{8 u^{3} Q_{c}}\left(\partial T_{q}\right)^{2}+\frac{c_{10}}{4 u^{3} Q_{c}}\left(\partial h_{q}\right) \cdot\left(\partial T_{q}\right)+\frac{c_{11}}{8 u^{3} Q_{c}}\left(\partial h_{q}\right)^{2}\right],(\mathrm{B} .9
\end{aligned}
$$

where the coefficients $\left\{c_{i}\right\}$ are given by

$$
\begin{aligned}
c_{1} & =\left(\frac{V_{c}^{\prime}}{V_{c}}\right)^{\prime}\left(1-\frac{T_{c}^{\prime 2}}{d_{c}}\right) \sqrt{d_{c}}+2 \frac{V_{c}^{\prime}}{V_{c}} \frac{h_{c}^{2} T_{c}}{\sqrt{d_{c}}}+\frac{h_{c}^{2}}{\sqrt{d_{c}}}+\frac{T_{c}^{\prime}}{\sqrt{d_{c}}}\left(\frac{h_{c}^{2} T_{c}}{d_{c}}\right)^{\prime} \\
c_{2} & =\left(\frac{h_{c} T_{c}^{2}}{d_{c}}\right)^{\prime} \frac{\frac{1}{4} f u^{3 / 2} h_{c}^{\prime}}{\sqrt{d_{c}}}+\frac{T_{c}^{2}}{\sqrt{d_{c}}} \\
c_{3} & =\frac{1}{\sqrt{d_{c}}}\left(1-\frac{\frac{1}{4} f u^{3 / 2} h_{c}^{\prime 2}}{d_{c}}\right) \frac{1}{4} f u^{3 / 2}, \\
c_{4}= & \frac{1}{\sqrt{d_{c}}}\left(1-\frac{T_{c}^{\prime 2}}{d_{c}}\right), \\
c_{5}= & \frac{V_{c}^{\prime}}{V_{c}} \frac{h_{c} T_{c}^{2}}{\sqrt{d_{c}}}+\left(2-\frac{h_{c}^{2} T_{c}^{2}}{d_{c}}\right) \frac{h_{c} T_{c}}{\sqrt{d_{c}}} \\
c_{6}= & -\frac{T_{c}^{\prime}}{d_{c} \sqrt{d_{c}}} \frac{1}{4} f u^{3 / 2} h_{c}^{\prime},
\end{aligned}
$$




$$
\begin{aligned}
c_{7} & =\frac{1}{\sqrt{d_{c}}}\left(\frac{V_{c}^{\prime}}{V_{c}}-\frac{h_{c}^{2} T_{c}}{d_{c}}\right) \frac{1}{4} f u^{3 / 2} h_{c}^{\prime}, \\
c_{8} & =-\frac{h_{c} T_{c}^{2} T_{c}^{\prime}}{d_{c} \sqrt{d_{c}}} \\
c_{9} & =4 u^{3 / 2} \sqrt{d_{c}}\left(1-\frac{T_{c}^{\prime 2}}{d_{c}}\right), \\
c_{10} & =-u^{3} \frac{f}{\sqrt{d_{c}}} h_{c}^{\prime} T_{c}^{\prime}, \\
c_{11} & =u^{3} f \sqrt{d_{c}}\left(1-\frac{\frac{1}{4} f u^{3 / 2} h_{c}^{\prime 2}}{d_{c}}\right),
\end{aligned}
$$

with $Q_{c}=\left(1+f u^{3 / 2} h_{c}^{2} T_{c}^{2}\right)$. For later convenience, we have explicitly written out a factor of $1 / 4 u^{3} Q_{c}$ in the coefficients in the last three terms in (B.9).

This action mixes $T_{q}$ and $h_{q}$ and the equations of motion derived from it reflect this mixing. After some manipulations, the equations can be cast in the form

$$
\begin{aligned}
& \partial^{2} T_{q}=a_{1} T_{q}+a_{2} T_{q}^{\prime}+a_{3} T_{q}^{\prime \prime}+a_{4} h_{q}+a_{5} h_{q}^{\prime}, \\
& \partial^{2} h_{q}=b_{1} h_{q}+b_{2} h_{q}^{\prime}+b_{3} h_{q}^{\prime \prime}+b_{4} T_{q}+b_{5} T_{q}^{\prime},
\end{aligned}
$$

where the coefficients $\left\{a_{i}\right\}$ and $\left\{b_{i}\right\}$ are given by

$$
\begin{array}{ll}
a_{1}=c_{10}\left(\overline{c_{7}}-c_{5}\right)+c_{11} c_{1}, & a_{2}=c_{10}\left(\overline{c_{6}}+c_{7}-c_{8}\right)-c_{11} \overline{c_{4}}, \quad a_{3}=c_{10} c_{6}-c_{11} c_{4}, \\
a_{4}=-c_{10} c_{2}+c_{11}\left(c_{5}-\overline{c_{8}}\right), & a_{5}=c_{10} \overline{c_{3}}-c_{11}\left(\overline{c_{6}}-c_{7}+c_{8}\right),
\end{array}
$$

and

$$
\begin{array}{ll}
b_{1}=c_{10}\left(\overline{c_{8}}-c_{5}\right)+c_{9} c_{2}, & b_{2}=c_{10}\left(\overline{c_{6}}-c_{7}+c_{8}\right)-c_{9} \overline{c_{3}}, \quad b_{3}=c_{10} c_{6}-c_{9} c_{3}, \\
b_{4}=-c_{10} c_{1}+c_{9}\left(c_{5}-\overline{c_{7}}\right), & b_{5}=c_{10} \overline{c_{4}}-c_{9}\left(\overline{c_{6}}+c_{7}-c_{8}\right) .
\end{array}
$$

Here we have used the notation $\overline{c_{i}}=\left(A c_{i}\right)^{\prime} / A$. As usual, a prime denotes a derivative with respect to $u$. Moreover, $\partial^{2}=\left(-\partial_{t}^{2}+\partial_{\vec{x}}^{2}\right)$ is the flat space-time laplacian. A possible term proportional to $h_{q}^{\prime \prime}$ is not present in (B.21) because its coefficient, $\left(c_{10} c_{3}-c_{11} c_{6}\right)$, vanishes. Similarly, in $(\mathbb{B} .22)$ the term proportional to $T_{q}^{\prime \prime}$ is absent because its coefficient, $\left(c_{10} c_{4}-c_{9} c_{6}\right)$, vanishes.

The equations of motion derived from (B.9) are quite complicated in general, but they simplify in the two asymptotic regimes of $u$.

$u \rightarrow u_{\max }$ : In this limit, many of the $c_{i}$ are small because they have at least one $\overline{\text { factor of } T_{c}}$ or its derivatives in them. The exceptions are $c_{1} \sim h_{0}^{2} u^{3 / 4}, c_{3} \sim$ $u^{9 / 4} / 4, c_{4} \sim u^{3 / 4}, c_{9} \sim 4 u^{3 / 4}$ and $c_{11} \sim u^{9 / 4}$. Retaining only the dominant terms in the equations, we get

$$
\begin{aligned}
-\left(u^{4} T_{q}^{\prime}(u, x)\right)^{\prime}+ & h_{0}^{2} u^{4} T_{q}(u, x)=0, \\
\left(u^{\frac{11}{2}} h_{q}^{\prime}(u, x)\right)^{\prime} & =0 .
\end{aligned}
$$


The term involving space-time laplacian on the fluctuations can be consistently neglected at the leading order since it is non-leading in powers of $u$, as can be verified a posteriori. These equations are identical to (2.15) and (2.17) and so have solutions similar to (2.16) and (2.18), but now with parameters that are functions of $x$ :

$$
\begin{aligned}
& T_{q}(u, x)=\frac{1}{u^{2}}\left(T_{q+}(x) e^{-h_{0} u}+T_{q-}(x) e^{h_{0} u}\right), \\
& h_{q}(u, x)=h_{q 0}(x)-h_{q 1}(x) u^{-9 / 2} .
\end{aligned}
$$

$u \rightarrow u_{0}$ : This limit is more involved, requiring a more detailed analysis. One expands $T_{q}$ and $h_{q}$ in powers of $\epsilon \equiv\left(u-u_{0}\right)$ with arbitrary $x$-dependent coefficients.

$$
\begin{aligned}
& T_{q}(u, x)=\frac{\sqrt{\pi}}{4} u_{0}^{3 / 2} \epsilon^{\omega}\left(\varphi_{0}(x)+\epsilon \varphi_{1}(x)+\cdots\right), \\
& h_{q}(u, x)=\sqrt{\frac{26}{\pi u_{0}}} u_{0}^{-3 / 4} \epsilon^{\tau}\left(\vartheta_{0}(x)+\epsilon \vartheta_{1}(x)+\cdots\right),
\end{aligned}
$$

Here, and in the following, we have set $f_{0}=1$. One also needs to expand the $a_{i}$ 's and $b_{i}$ 's in powers of $\epsilon$. Retaining up to the first nonleading power in $\epsilon$, we get

$$
\begin{aligned}
& a_{1}=8 \xi \epsilon^{-1}\left(1+\frac{23 \epsilon}{12 u_{0}}\right), \quad a_{2}=2 \xi\left(1+\frac{2 \epsilon}{u_{0}}\right), \quad a_{3}=\frac{4 u_{0}^{-3 / 2}}{\pi} \xi \epsilon^{3}\left(1+\frac{23 \epsilon}{12 u_{0}}\right), \\
& a_{4}=\frac{2 \pi u_{0}^{11 / 4}}{\sqrt{26}} \xi \epsilon^{-7 / 2}\left(1+\frac{65 \epsilon}{24 u_{0}}\right), \quad a_{5}=\frac{4 u_{0}^{5 / 4}}{\sqrt{26}} \xi \epsilon^{-1 / 2}\left(1+\frac{21 \epsilon}{8 u_{0}}\right),
\end{aligned}
$$

and

$$
\begin{aligned}
& b_{1}=-3 \xi \epsilon^{-1}\left(1+\frac{3 \epsilon}{4 u_{0}}\right), \quad b_{2}=2 \xi\left(1+\frac{2 \epsilon}{u_{0}}\right), \quad b_{3}=\frac{4 u_{0}^{-3 / 2}}{\pi} \xi \epsilon^{3}\left(1+\frac{23 \epsilon}{12 u_{0}}\right), \\
& b_{4}=\frac{16 \sqrt{26} u_{0}^{-11 / 4}}{\pi} \xi \epsilon^{3 / 2}\left(-1+\frac{\epsilon}{24 u_{0}}\right), \quad b_{5}=-\frac{4 \sqrt{26} u_{0}^{-11 / 4}}{\pi} \xi \epsilon^{5 / 2}\left(1+\frac{\epsilon}{24 u_{0}}\right),
\end{aligned}
$$

where $\xi=-13 u_{0}^{2} / 8$. Substituting these expansions in the equations (B.21), (B.22) and comparing different orders of $\epsilon$, we see that a consistent solution exists only for $\omega=-3$ and $\tau=-1 / 2$, and then we get

$$
\vartheta_{0}(x)=-\frac{1}{4} \varphi_{0}(x), \quad \varphi_{1}(x)=\frac{5}{6 u_{0}} \varphi_{0}(x), \quad \vartheta_{1}(x)=\frac{1}{8 \xi}\left(\partial^{2}+\frac{65 u_{0}}{32}\right) \varphi_{0}(x) .
$$

The first of these relations is precisely what is needed to think of the leading terms in (B.28) as coming from expanding $\left(u-u_{0}(x)\right)^{-1}$ around a constant $u_{0}$. The last relation shows that when $x$-dependence is allowed, not all coefficients get uniquely determined. In fact, the part of $\varphi_{0}(x)$ annihilated by the operator on the right hand side does not show up in $\vartheta_{1}(x)$.

The above analysis shows that perturbation expansion in "small" fluctuations around a constant $u_{0}$ is singular, although we have obtained a solution by a formal expansion. 


\section{Calculation of the exact $(u, x)$-dependent action}

This involves calculating the determinant of the matrix $(1+K)$, whose elements are given in (4.2). We will simplify this calculation by making use of the following trick. Consider the family of determinants, $D(\lambda) \equiv \operatorname{det}(1+\lambda K)$, where $\lambda$ is an arbitrary parameter. We actually only need to calculate $D(1)$, but this calculation can be reduced essentially to the calculation of the inverse of the matrix $(1+\lambda K)$, which turns out to be much easier than a direct computation of the determinant. Consider the following:

$$
\frac{d}{d \lambda} D(\lambda)=D(\lambda) \operatorname{tr}\left[(1+\lambda K)^{-1} K\right]
$$

We can obtain $\Delta$ by integrating this equation, using the boundary condition $D(0)=1$ :

$$
\ln D(1)=\int_{0}^{1} d \lambda D(\lambda)^{-1} \frac{d}{d \lambda} D(\lambda)=\int_{0}^{1} d \lambda \operatorname{tr}\left[(1+\lambda K)^{-1} K\right]
$$

This reduces the required calculation to finding the inverse matrix $M(\lambda)=(1+$ $\lambda K)^{-1}$, which may be done as follows. Using the defining equation, $(1+\lambda K) M(\lambda)=$ 1 , one can express all components of $M$ in terms of $M^{\mu}{ }_{\nu}$ :

$$
M_{\nu}^{u}=-\lambda K_{\mu}^{u} M_{\nu}^{\mu}, \quad M^{u}{ }_{u}=1-\lambda^{2} K_{\mu}^{u} K^{\nu}{ }_{u} M^{\mu}{ }_{\nu}, \quad M^{\mu}{ }_{u}=-\lambda M^{\mu}{ }_{\nu} K^{\nu}{ }_{u} .
$$

Moreover, one can show that $M^{\mu}{ }_{\nu}$ satisfies

$$
P^{\mu}{ }_{\sigma} M^{\sigma}{ }_{\nu}=\delta^{\mu}{ }_{\nu}, \quad P^{\mu}{ }_{\sigma} \equiv\left(\delta^{\mu}{ }_{\sigma}+\lambda K^{\mu}{ }_{\sigma}-\lambda^{2} K^{\mu}{ }_{u} K^{u}{ }_{\sigma}\right) .
$$

Thus, to find $M(\lambda)$ we need to find the inverse of the $P^{\mu}{ }_{\sigma}(\lambda)$ matrix. First note that using (4.2) we can write

$$
P_{\sigma}^{\mu}(\lambda)=\delta^{\mu}{ }_{\nu}+\beta_{1}(\lambda) \partial^{\mu} T \partial_{\nu} T+\beta_{2}(\lambda) \partial^{\mu} h \partial_{\nu} h+\beta_{3}(\lambda)\left(\partial^{\mu} T \partial_{\nu} h+\partial^{\mu} h \partial_{\nu} T\right),
$$

where

$$
\begin{array}{ll}
\beta_{1}(\lambda)=\frac{\lambda u^{-3 / 2}}{Q}\left(1-\lambda \frac{T^{\prime 2}}{d_{T}}\right), & \beta_{2}(\lambda)=\frac{\lambda f}{4 Q}\left(1-\lambda \frac{f u^{3 / 2} h^{\prime 2}}{4 d_{T}}\right), \\
\beta_{3}(\lambda)=-\frac{\lambda^{2} f h^{\prime} T^{\prime}}{4 Q d_{T}}, & \beta_{4}(\lambda)=\beta_{1}(\lambda) \beta_{2}(\lambda)-\left(\beta_{3}(\lambda)\right)^{2} .
\end{array}
$$

For $\lambda=1$ these reduce to the $\beta$ 's in (4.5). Now, from the general structure of the $P^{\mu}{ }_{\nu}$ matrix, we can parametrize the $M^{\mu}{ }_{\nu}$ matrix as

$$
M^{\mu}{ }_{\nu}(\lambda)=\delta^{\mu}{ }_{\nu}+\alpha_{1}(\lambda) \partial^{\mu} T \partial_{\nu} T+\alpha_{2}(\lambda) \partial^{\mu} h \partial_{\nu} h+\alpha_{3}(\lambda)\left(\partial^{\mu} T \partial_{\nu} h+\partial^{\mu} h \partial_{\nu} T\right) .
$$


We have calculated the $\alpha$ 's. They work out to be

$$
\begin{aligned}
& \alpha_{1}(\lambda)=-\frac{1}{\Delta(\lambda)}\left[\beta_{1}(\lambda)+\beta_{4}(\lambda)(\partial h)^{2}\right] \\
& \alpha_{2}(\lambda)=-\frac{1}{\Delta(\lambda)}\left[\beta_{2}(\lambda)+\beta_{4}(\lambda)(\partial T)^{2}\right] \\
& \alpha_{3}(\lambda)=-\frac{1}{\Delta(\lambda)}\left[\beta_{3}(\lambda)-\beta_{4}(\lambda) \partial h . \partial T\right]
\end{aligned}
$$

Here $\Delta(\lambda)$ is a generalization of $\Delta$ defined in (4.4). It has the same form but with the above $\lambda$-dependent $\beta$ 's replacing those in (4.4). By definition, $\Delta(1)=\Delta$.

Armed with the inverse matrix $M(\lambda)$, we can now compute the trace on the right hand side of (C.2). Using (C.3) and (4.2), we first note that

$$
\operatorname{tr}\left[(1+\lambda K)^{-1} K\right]=M_{\sigma}^{\mu}(\lambda) \frac{d}{d \lambda} P_{\mu}^{\sigma}(\lambda) .
$$

Given the equations (C.5)-(C.8), it is straightforward, though tedious, to compute the right hand side of the above equation. One gets the simple result

$$
M_{\sigma}^{\mu}(\lambda) \frac{d}{d \lambda} P_{\mu}^{\sigma}(\lambda)=\Delta(\lambda)^{-1} \frac{d}{d \lambda} \Delta(\lambda) \text {. }
$$

It follows from this and (C.2) that $D(1)=\Delta(1)=\Delta$. Hence the complete 5dimensional action is that given in (4.3).

To compute the equations of motion for $T(u, x)$ and $h(u, x)$ that follow from this action, we will need the following, which can be easily calculated from the relation $\Delta_{T}=d_{T} \Delta$ and the definition of $\Delta$ given in (4.4):

$$
\begin{aligned}
& \frac{1}{2} \frac{\partial \Delta_{T}}{\partial T^{\prime}}=T^{\prime}+\frac{f T^{\prime}}{4 Q}(\partial h)^{2}-\frac{f h^{\prime}}{4 Q} \partial T . \partial h \\
& \frac{1}{2} \frac{\partial \Delta_{T}}{\partial\left(\partial_{\mu} T\right)}=d_{T} \beta_{1} \partial^{\mu} T+d_{T} \beta_{3} \partial^{\mu} h+\frac{u^{-3}}{4 Q}\left(\partial^{\mu} T(\partial h)^{2}-\partial^{\mu} h(\partial h . \partial T)\right) \\
& \frac{1}{2} \frac{\partial \Delta_{T}}{\partial T}=T h^{2}\left[1-\frac{f^{2} u^{3 / 2}}{4 Q^{2}}\left(h^{\prime 2}(\partial T)^{2}+T^{\prime 2}(\partial h)^{2}-2 T^{\prime} h^{\prime}(\partial T . \partial h)\right.\right. \\
&\left.\left.\quad+f^{-1} u^{-3}\left((\partial T)^{2}(\partial h)^{2}-(\partial T . \partial h)^{2}\right)\right)\right] \\
& \frac{1}{2} \frac{\partial \Delta_{T}}{\partial h^{\prime}}=\frac{f u^{3 / 2}}{4\left(\partial_{\mu} h\right)}=d_{T} \beta_{2} \partial^{\mu} h+\frac{f h^{\prime}}{4 Q}(\partial T)^{2}-\frac{f T^{\prime}}{4 Q} \partial T . \partial h, \\
& \frac{1}{2} \frac{\partial \Delta_{T}}{\partial h}=T^{2} h\left[1-\frac{f^{2} u^{3 / 2}}{4 Q^{2}}\left(h^{\prime 2}(\partial T)^{2}+T^{\prime 2}(\partial h)^{2}-2 T^{\prime} h^{\prime}(\partial T . \partial h)\right.\right. \\
&\left.\left.\quad+f^{-1} u^{-3}\left((\partial T)^{2}(\partial h)^{2}-(\partial T . \partial h)^{2}\right)\right)\right] .
\end{aligned}
$$


Using these one can show that

$$
\begin{aligned}
& \Delta_{T}- T^{\prime} \frac{1}{2} \frac{\partial \Delta_{T}}{\partial T^{\prime}}-\partial_{\mu} T \frac{1}{2} \frac{\partial \Delta_{T}}{\partial\left(\partial_{\mu} T\right)}=d_{T}-T^{\prime 2}+\frac{u^{-3 / 2}}{4}(\partial h)^{2}, \\
& T^{\prime} \frac{1}{2} \frac{\partial \Delta_{T}}{\partial h^{\prime}}+\partial_{\mu} T \frac{1}{2} \frac{\partial \Delta_{T}}{\partial\left(\partial_{\mu} h\right)}=\frac{f u^{3 / 2}}{4} T^{\prime} h^{\prime}+\frac{u^{-3 / 2}}{4}(\partial T . \partial h) .
\end{aligned}
$$

We can now give the equations of motion obtained from the action (4.3):

$$
\begin{gathered}
\frac{u^{13 / 4}}{\sqrt{\Delta_{T}}}\left[\frac{1}{2} \frac{\partial \Delta_{T}}{\partial T}+\frac{V^{\prime}}{V}\left(d_{T}-T^{\prime 2}+\frac{u^{-3 / 2}}{4}(\partial h)^{2}\right)\right] \\
=\left(\frac{u^{13 / 4}}{\sqrt{\Delta_{T}}} \frac{1}{2} \frac{\partial \Delta_{T}}{\partial T^{\prime}}\right)^{\prime}+\partial_{\mu}\left(\frac{u^{13 / 4}}{\sqrt{\Delta_{T}}} \frac{1}{2} \frac{\partial \Delta_{T}}{\partial\left(\partial_{\mu} T\right)}\right) \\
\frac{u^{13 / 4}}{\sqrt{\Delta_{T}}}\left[\frac{1}{2} \frac{\partial \Delta_{T}}{\partial h}-\frac{V^{\prime}}{V}\left(\frac{f u^{3 / 2}}{4} T^{\prime} h^{\prime}+\frac{u^{-3 / 2}}{4}(\partial T . \partial h)\right)\right] \\
=\left(\frac{u^{13 / 4}}{\sqrt{\Delta_{T}}} \frac{1}{2} \frac{\partial \Delta_{T}}{\partial h^{\prime}}\right)^{\prime}+\partial_{\mu}\left(\frac{u^{13 / 4}}{\sqrt{\Delta_{T}}} \frac{1}{2} \frac{\partial \Delta_{T}}{\partial\left(\partial_{\mu} h\right)}\right)
\end{gathered}
$$

These can be further simplified using the expressions given in (C.11), but we will not do so here since we will only be interested in a leading solution to these equations in the limit $u \sim u_{0}$. As a check, we note that these equations reduce to the equations (2.13) and (2.14) if $T$ and $h$ are $x$-independent.

\section{References}

[1] T. Sakai and S. Sugimoto, Low energy hadron physics in holographic QCD, Prog. Theor. Phys. 113, 843 (2005), hep-th/0412141.

[2] E. Witten, Anti-de Sitter space, thermal phase transitions, and confinement in gauge theories, Adv. Theor. Math. Phys. 2, 505 (1998), hep-th/9803131.

[3] A. Karch and A. Katz, Adding flavour to AdS/CFT, JHEP 0206 (2002) 043, hep-th/0205236.

[4] J. Babington, J. Erdmenger, N. J. Evans, Z. Guralnik and I. Kirsch, Chiral symmetry breaking and pions in non-supersymmetric gauge/gravity duals, Phys. Rev. D 69 (2004) 066007, hep-th/0306018.

[5] M. Kruczenski, D. Mateos, R. C. Myers and D. J. Winters, Towards a holographic dual of large-N(c) QCD, JHEP 0405 (2004) 041, hep-th/0311270.

[6] T. Sakai and J. Sonnenschein, Probing flavoured mesons of confining gauge theories by supergravity, JHEP 0309 (2003) 047, hep-th/0305049.

[7] J. L. F. Barbon, C. Hoyos, D. Mateos and R. C. Myers, The holographic life of the eta', JHEP 0410, 029 (2004), hep-th/0404260. 
[8] H. Nastase, On Dp-Dp+4 systems, QCD dual and phenomenology, hep-th/0305069.

[9] T. Sakai and S. Sugimoto, More on a holographic dual of QCD, Prog. Theor. Phys. 114, 1083 (2006), hep-th/0507073.

[10] H. Hata, T. Sakai and S. Sugimoto, Baryons from instantons in holographic QCD, hep-th/0701280.

[11] D. K. Hong, M. Rho, H. U. Yee and P. Yi, Chiral dynamics of baryons from string theory, hep-th/0701276.

[12] K. Nawa, H. Suganuma and T. Kojo, Brane-induced Skyrmions: Baryons in holographic QCD, hep-th/0701007.

[13] O. Bergman, G. Lifschytz and M. Lippert, Holographic nuclear physics, arXiv:0708.0326.

[14] D. Yamada, Sakai-Sugimoto model at high density, arXiv:0707.0101.

[15] O. Aharony, J. Sonnenschein and S. Yankielowicz, A holographic model of deconfinement and chiral symmetry restoration, Annals Phys. 322 (2007) 1420, hep-th/0604161.

[16] E. Antonyan, J. A. Harvey, S. Jensen and D. Kutasov, NJL and QCD from string theory, hep-th/0604017.

[17] A. Parnachev and D. A. Sahakyan, Chiral phase transition from string theory, Phys. Rev. Lett. 97 (2006) 111601, hep-th/0604173.

[18] A. Sen, Tachyon dynamics in open string theory, Int. J. Mod. Phys. A 20 (2005) 5513 , hep-th/0410103.

[19] R. Casero, E. Kiritsis and A. Paredes, Chiral symmetry breaking as open string tachyon condensation, Nucl. Phys. B787 (2007) 98, hep-th/0702155.

[20] O. Bergmann, S. Seki and J. Sonnenschein, Quark mass and condensate in HQCD, JHEP 0712 (2007) 037, arXiv:0708.2839.

[21] A. Dhar and P. Nag, Sakai-Sugimoto model, Tachyon Condensation and Chiral symmetry Breaking, JHEP 0801 (2008) 055, arXiv:0708.3233.

[22] E. Witten, Anti-de Sitter space and holography, Adv. Theor. Math. Phys. 2, 253 (1998), hep-th/9802150.

[23] V. Balasubramanian, P. Kraus and A. E. Lawrence Bulk versus boundary dynamics in anti-de Sitter space-time, Phys. Rev. D59 (1999) 046003, hep-th/9805171.

[24] V. Balasubramanian, P. Kraus, A. E. Lawrence and S. P. Trivedi, Holographic probes of anti-de Sitter space-times, Phys. Rev. D59 (1999) 104021, hep-th/9808017. 
[25] O. Aharony, S. S. Gubser, J. M. Maldacena, H. Ooguri and Y. Oz, Large N field theories, string theory and gravity, Phys. Rept. 323 (2000) 183, hep-th/9905111.

[26] S. Sugimoto and K. Takahashi, QED and String Theory, Adv. Theor. Math. Phys. 3 (1999) 281, hep-th/0403247.

[27] N. Itzhaki, J. M. Maldacena, J. Sonnenschein and S. Yankielowicz, Supergravity and the large $N$ limit of theories with sixteen supercharges, Phys. Rev. D 58 (1998) 046004, hep-th/9802042.

[28] O. Aharony and D. Kutasov, Holographic Duals of Long Open Strings, arXiv:0803.3547.

[29] K. Hashimoto, T. Hirayama, F. Lin and H. Yee, Quark Mass Deformation of Holographic Massless QCD, arXiv:0803.4192.

[30] A. A. Tseytlin, On non-abelian generalization of the Born-Infeld action in string theory, Nucl. Phys. B 501 (1997) 41, hep-th/9701125.

[31] A. Sen, Dirac-Born-Infeld action on the tachyon kink and vortex, Phys. Rev. D 68 (2003) 066008, hep-th/0303057.

[32] M. R. Garousi, D-brane anti-D-brane effective action and brane interaction in open string channel, JHEP 0501 (2005) 029, hep-th/0411222.

[33] K. B. Fadafan and M. R. Garousi, Non-abelian expansion of S-matrix elements and non-abelian tachyon DBI action, hep-th/0607249; M. R. Garousi, On the effective action of D-brane-anti-D-brane system, arXiv:0710.5469; M. R. Garousi and H. Golchin, On higher derivative corrections of the tachyon action, arXiv:0801.3358; M. R. Garousi and E. Hatefi, On Wess-Zumino terms of Brane-Antibrane systems, arXiv:0710.5875. M. R. Garousi, Higher derivative corrections to Wess-Zumino action of Brane-Antibrane systems, arXiv:0712.1954.

[34] C. j. Kim, H. B. Kim, Y. b. Kim and O. K. Kwon, Electromagnetic string fluid in rolling tachyon, JHEP 0303 (2003) 008, hep-th/0301076.

[35] F. Leblond and A. W. Peet, SD-brane, gravity fields and rolling tachyons, JHEP 0304 (2003) 048, hep-th/0303035.

[36] N. Lambert, H. Liu and J. M. Maldacena, Closed strings from decaying D-branes, hep-th/0303139.

[37] J. A. Minahan and B. Zwiebach, Effective tachyon dynamics in superstring theory, JHEP 0103 (2001) 038, hep-th/0009246.

[38] D. Kutasov, M. Marino and G. W. Moore, Remarks on tachyon condensation in superstring field theory, hep-th/0010108.

[39] P. Kraus and F. Larsen, Boundary string field theory of the DD-bar system, Phys. Rev. D63 (2001) 106004, hep-th/0012198. 
[40] T. Takayanagi, S. Terashima and T. Uesugi, Brane-antibrane action from boundary string field theory, JHEP 0103 (2001) 019, hep-th/0012210.

[41] M. Bianchi, D. Z. Freedman and K. Skenderis, Holographic renormalization, Nucl. Phys. B631 (2002) 159, hep-th/0112119.

[42] K. Skenderis, Lecture Notes on Holographic Renormalization, Class. Quant. Grav. 19 (2002) 5849, hep-th/0209067.

[43] A. Karch, A. O'Bannon and K. Skenderis, Holographic Renormalization of Probe D-Branes in AdS/CFT, JHEP 0604 (2006) 015, hep-th/0512125.

[44] J. Erdmenger, N. Evans, I. Kirsch and E. Threlfall, Mesons in Gauge/Gravity Duals - A Review, arXiv:0711.4467.

[45] J. Erlich, E. Katz, D. T. Son and M. A. Stephanov, QCD and a holographic model of hadrons, Phys. Rev. Lett. 95 (2005) 261602, hep-ph/0501128.

[46] L. Da Rold and A. Pomarol Chiral symmetry breaking from five dimensional spaces, Nucl. Phys. B721 (2005) 79, hep-ph/0501218. 